\title{
Effect of the electron motion on the Compton scattering of a twisted photon
}

\author{
Joseph A. Sherwin $\mathbb{0}^{*}$ \\ Department of Physics, Metropolitan Community College, Omaha, Nebraska 68103-0777, USA
}

(Received 18 October 2019; revised manuscript received 18 December 2019; accepted 24 January 2020; published 19 February 2020)

\begin{abstract}
We present theoretical and numerical results on the Compton scattering of a twisted Bessel photon by a plane-wave electron with an arbitrary initial velocity. Scattering of a photon prepared in a superposition of Bessel states is also explored. Specifically, we investigate how the electron motion affects the dependence of the angular distribution and polarization of the scattered photons on the twisted photon opening angle, projection of angular momentum, and on the superposition of angular-momentum projection. The motion of the electron is found to strongly influence the dependence of these quantities on the parameters of the twisted photon. Moreover, if the speed of a properly directed electron exceeds a critical value, various distributions, such as the degree of circular polarization of the scattered photons, undergo a nearly global reversal of sign that is absent from the scattering of a plane-wave photon under identical conditions. This behavior is found to correspond to the inversion of the twisted photon momentum cone in the rest frame of the electron.
\end{abstract}

DOI: 10.1103/PhysRevResearch.2.013168

\section{INTRODUCTION}

An optical vortex, or twisted light, unlike an ordinary plane wave, is an electromagnetic wave with a helical wavefront and an azimuthally varying phase about its central axis of propagation [1-4]; the corresponding quantized excitation is a so-called twisted photon. Remarkably, besides the conventional spin angular momentum (SAM), twisted light can possess an intrinsic orbital angular momentum (OAM) along its direction of propagation [5-7], which can be used, for example, in optical communications [8,9], the manipulation of microparticles [10,11], and microscopy [12]. Furthermore, recent theoretical studies report on the effects of using twisted light on the photoexcitation of hydrogenlike atoms and ions [13-15], many electron atoms and ions [16,17], and atomic spectroscopy [18]. Recent experiments [19-21] on the photoexcitation of trapped calcium ions strongly support many of these predictions (e.g., modified, position-dependent selection rules).

There are also theoretical predictions on twisted light in the extreme ultraviolet, x-ray, and gamma-ray frequencies. Several representative studies include such diverse phenomena as photoionization [22], Rayleigh scattering [23], Thomson and Compton scattering [24-26], double Compton scattering [27], photodisintegration of deuteron [28], and photonuclear reactions [29].

The study of these and other high-energy phenomena will likely require sources quite different from those suitable for

\footnotetext{
*jsherwin99@hotmail.com

Published by the American Physical Society under the terms of the Creative Commons Attribution 4.0 International license. Further distribution of this work must maintain attribution to the author(s) and the published article's title, journal citation, and DOI.
}

the generation of twisted light in the optical frequency range. Progress has been made toward the ultraviolet and extreme ultraviolet ranges by utilizing high harmonic generation [30,31] which has also been observed recently [32]. Possible sources of twisted photons in the $\mathrm{MeV}$ and $\mathrm{GeV}$ ranges include the use of undulators and undulator-type devices [33-35], the upconversion of low-energy twisted photons through inverse Compton scattering [36-38], plasma-wakefield acceleration [39], and nonlinear inverse Thomson scattering [29,40] of intense circularly polarized light.

In particular, it is pointed out in Refs. [29,40] that the generation of twisted photons by nonlinear inverse Thomson scattering can be realized in various astrophysical environments where intense electromagnetic waves and highenergy electrons coexist, such as in the vicinity of magnetized neutron stars and the fronts of relativistic shocks. Also, it is thought that plane-wave photons can be converted into twisted photons in the vicinity of Kerr black holes [41]. Therefore high-energy twisted photons may be quite common throughout the universe. Moreover, they will likely coexist with high-energy electrons, which may collide with them. Alternately, as twisted gamma photons become readily available in terrestrial laboratories, their interaction with electrons and other particles will be explored over a wide range of their parameter space. It is important, therefore, to examine Compton scattering of a twisted photon not only from an electron at rest [24,25], or for special cases [36,37], but also for general scenarios in which an electron is in motion.

In this paper, we examine the degree to which the motion of a plane-wave electron influences the dependence of the angular distributions and polarization of the Compton scattered photons on the projection of total angular momentum and opening angle of an incident twisted Bessel photon and compare these results to those corresponding to an incident plane-wave photon. The organization of the paper is as follows. In Sec. II, we develop the theoretical formalism to 


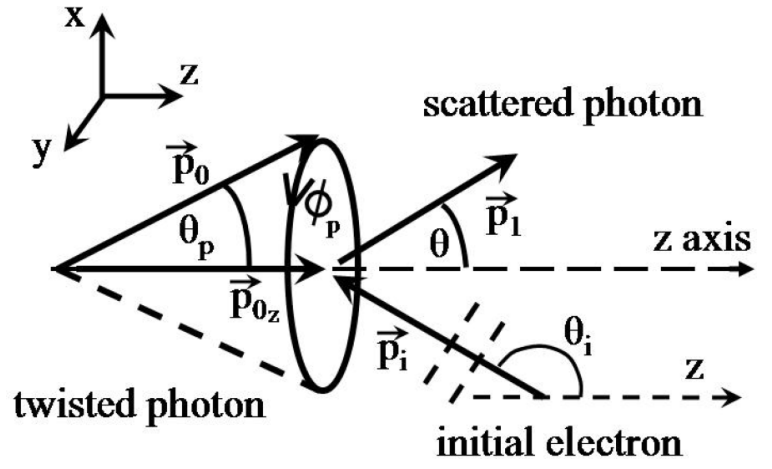

FIG. 1. Compton scattering of an incoming twisted photon from a plane-wave electron.

calculate the cross sections and Stokes parameters for Compton scattering of a twisted Bessel photon from a plane-wave electron moving with arbitrary velocity. In Sec. III, calculations of the angular distributions of these quantities for Compton scattering of a twisted gamma photon are presented for several choices of the electron speed and direction of motion and compared with corresponding results for an incident plane-wave photon. This is followed by results on the Compton scattering of a photon prepared in a superposition of two twisted Bessel states. Finally, in Sec. IV, a summary of the main results and conclusions is given. Throughout the paper, we use units of rationalized meters-kilograms-seconds and a space-time metric of $g^{\mu \nu}=\operatorname{diag}(1,-1,-1,-1)$. The scalar product between two 4 -vectors is denoted by $a \cdot b=a^{\mu} b_{\mu}=$ $a^{0} b^{0}-\vec{a} \cdot \vec{b}$, consistent with the choice of space-time metric, and contraction of the Dirac matrices $\gamma^{\mu}$ with a 4-vector $a$ is given by $\hat{a}=\gamma^{\mu} a_{\mu}$.

\section{THEORY AND BACKGROUND}

\section{A. Description of the problem}

The scattering interaction is illustrated in Fig. 1. A twisted Bessel photon with opening angle $\theta_{p}$ and total energy $E_{0}$ propagates in the positive $z$ direction and scatters off of a plane-wave electron with well-defined momentum $\vec{p}_{i}$, total energy $E_{i}$, and kinetic energy $T_{i}=E_{i}-m_{e} c^{2}$. The scattered photon is resolved into a plane-wave state with linear momentum $\vec{p}_{1}$. The scattered electron has linear momentum $\vec{p}_{f}$. This scenario is a so-called single-vortex or $\mathrm{V}+\mathrm{PW} \rightarrow \mathrm{PW}+$ PW scattering process [42], where $\mathrm{V}$ designates a twisted (vortex) particle, and PW designates a plane-wave particle.

Only the projection of the incident twisted photon momentum onto its average direction of propagation is well defined, which here coincides with the positive $z$ direction. The component of momentum along this direction is designated $p_{0 z}=\kappa_{p \|}$, where $\kappa_{p \|}$ is the longitudinal momentum quantum number; this direction also defines the projection of total angular momentum projection of the twisted photon. The transverse component its momentum-here the component projected into the $x-y$ plane-has indeterminate direction but has well defined magnitude $\left|\vec{p}_{0_{\perp}}\right|=\kappa_{p_{\perp}}$, where $\kappa_{p_{\perp}}$ is the transverse momentum quantum number. Therefore the momentum vectors of the plane-wave states making up the twisted Bessel state sweep out a cone of fixed opening angle $\theta_{p}$, given by $\tan \theta_{p}=\kappa_{p_{\perp}} / \kappa_{p_{\|}}$, with transverse component specified through the azimuthal angle $\phi_{p}$. In the following section, a precise mathematical description of the twisted Bessel state will be given.

The momentum 4-vector of the scattered photon or a planewave component of the incident twisted photon are given by $p_{j}=\hbar k_{j}$, with $k_{j}=(\omega / c) n_{j}$, and where

$$
n_{j}=\left(1, \vec{n}_{j}\right)=\left(1, \sin \theta_{j} \cos \phi_{j}, \sin \theta_{j} \sin \phi_{j}, \cos \theta_{j}\right),
$$

is a null 4-vector. The variables $\phi_{j}$ and $\theta_{j}(j=0,1)$ determine the azimuthal and the polar angles of the unit 3-vector $\vec{n}_{j}$. Here, $j=0$ denotes the incident photon (in which case $\theta_{0}=$ $\theta_{p}$ and $\left.\phi_{0}=\phi_{p}\right)$, and $j=1$ denotes the scattered photon.

The 4-momentum of the initial electron is $p_{i}=\left(E_{i} / c, \vec{p}_{i}\right)$ with the 3-momentum written as $\vec{p}_{i}=\left|\vec{p}_{i}\right| \vec{n}_{i}$. The Cartesian components of the unit 3 -vector $\vec{n}_{i}$ are given by

$$
\vec{n}_{i}=\left(\sin \theta_{i} \cos \phi_{i}, \sin \theta_{i} \sin \phi_{i}, \cos \theta_{i}\right),
$$

where $\phi_{i}$ and $\theta_{i}$ are the azimuthal and the polar angles of the initial-electron momentum, respectively.

The Stokes parameters, introduced later, require for their calculation several choices of polarization 4-vectors of the photons. One set is defined here according to the choice

$$
\begin{aligned}
\epsilon_{j}^{1} & =\left(0, \vec{\epsilon}_{j}^{1}\right)=\left(0, \cos \theta_{j} \cos \phi_{j}, \cos \theta_{j} \sin \phi_{j},-\sin \theta_{j}\right), \\
\epsilon_{j}^{2} & =\left(0, \vec{\epsilon}_{j}^{2}\right)=\left(0,-\sin \phi_{j}, \cos \phi_{j}, 0\right) .
\end{aligned}
$$

A second set of linear polarization vectors is defined in terms of the first set as

$$
\epsilon_{j}^{ \pm}=\frac{1}{\sqrt{2}}\left(\epsilon_{j}^{1} \pm \epsilon_{j}^{2}\right) .
$$

Finally, the helicity polarization 4 -vectors are given by

$$
\begin{aligned}
& \epsilon_{j}^{+1}=\frac{-1}{\sqrt{2}}\left(\epsilon_{j}^{1}+i \epsilon_{j}^{2}\right), \\
& \epsilon_{j}^{-1}=\frac{1}{\sqrt{2}}\left(\epsilon_{j}^{1}-i \epsilon_{j}^{2}\right) .
\end{aligned}
$$

In the following section, we briefly summarize the description of twisted Bessel states.

\section{B. Twisted-photon states and the twisted cross section}

A photon in a twisted Bessel state with definite helicity $\Lambda$ and total angular momentum (TAM) projection $m$ can be described by its decomposition into states of definite momentum and helicity $[24,36,43]$ according to

$$
\left|\kappa_{p_{\perp}} \kappa_{p_{\|}} m \Lambda\right\rangle=\int \frac{V d^{3} p_{0}}{(2 \pi \hbar)^{3}} b_{\kappa_{p_{\perp}} \kappa_{p_{\|}} m}\left(\vec{p}_{0}\right)\left|\vec{p}_{0} \Lambda\right\rangle,
$$

where $\kappa_{p_{\perp}}$ is the aforementioned transverse momentum quantum number, $\kappa_{p_{\|}}$is the longitudinal momentum quantum number, and the amplitude corresponding to a plane-wave component is

$$
b_{\kappa_{p_{\perp}} \kappa_{p_{\|}} m}\left(\vec{p}_{0}\right)=N_{\mathrm{tw}} \delta\left(p_{0_{z}}-\kappa_{p_{\|}}\right) a_{\kappa_{p_{\perp}} m}\left(\vec{p}_{0_{\perp}}\right),
$$

where the transverse amplitude $a_{\kappa_{\perp} m}\left(\vec{p}_{0_{\perp}}\right)$ is defined by

$$
a_{\kappa_{p_{\perp}} m}\left(\vec{p}_{0_{\perp}}\right)=\sqrt{\frac{2 \pi \hbar}{\kappa_{p_{\perp}}}}(-i)^{m} e^{i m \phi_{p}} \delta\left(\left|\vec{p}_{0_{\perp}}\right|-\kappa_{p_{\perp}}\right) .
$$


The normalization factor $N_{\mathrm{tw}}$ that appears in $b_{\kappa_{p_{\perp}} \kappa_{p_{\|}} m}\left(\vec{p}_{0}\right)$ is determined such that the twisted Bessel states satisfy the normalization [24]

$$
\begin{aligned}
& \left\langle\kappa_{p_{\perp}} \kappa_{p_{\|}} m \Lambda \mid \kappa_{p_{\perp}}^{\prime} \kappa_{p_{\|}}^{\prime} m^{\prime} \Lambda^{\prime}\right\rangle \\
& \quad=\frac{2 \pi^{2} \hbar^{2}}{R L_{z}} \delta_{m m^{\prime}} \delta_{\Lambda \Lambda^{\prime}} \delta\left(\kappa_{p_{\perp}}-\kappa_{p_{\perp}}^{\prime}\right) \delta\left(\kappa_{p_{\|}}-\kappa_{p_{\|}}^{\prime}\right) .
\end{aligned}
$$

This corresponds to a normalization of one particle per cylindrical volume $V=\pi R^{2} L_{z}$, where both radius $R$ and length $L_{z}$ are made infinitely large.

To establish an explicit connection between the twisted Bessel state described in Eq. (6) and the standard momentumspace formulation of quantum electrodynamics, a twisted $S$ matrix [36,37,43] for Compton scattering in a $\mathrm{V}+\mathrm{PW} \rightarrow$ $\mathrm{PW}+\mathrm{PW}$ process is defined as follows

$$
\begin{aligned}
S_{\mathrm{tw}} & =\left\langle p_{1} \Lambda_{1} ; p_{f} \lambda^{\prime}|S| \kappa_{p_{\perp}} \kappa_{p_{\|}} m \Lambda ; p_{i} \lambda\right\rangle, \\
& =\int \frac{V d^{3} p_{0}}{(2 \pi \hbar)^{3}} b_{\kappa_{p_{\perp}} \kappa_{p_{\|}} m}\left(\vec{p}_{0}\right) S_{\mathrm{pw}},
\end{aligned}
$$

where $S_{\mathrm{pw}}=\left\langle p_{1} \Lambda_{1} ; p_{f} \lambda^{\prime}|S| p_{0} \Lambda ; p_{i} \lambda\right\rangle$ is the standard planewave $S$ matrix formulated entirely in terms of particles of definite momenta. Here, $p_{i}$ is the 4-momentum of the initial electron, $p_{0}$ is the 4-momentum of the incident photon, $p_{1}$ is the 4-momentum of the scattered photon, while $p_{f}$ is the 4-momentum of the scattered electron, and $\Lambda_{1}$ is the helicity of the final plane-wave photon, while $\lambda, \lambda^{\prime}= \pm 1 / 2$ are the helicities of the initial and final electrons, respectively.

A corresponding twisted cross section $d \sigma_{\mathrm{tw}}$ can be developed from the twisted $S$ matrix and is related to the cross section involving only plane-wave particles $d \sigma_{\mathrm{pw}}$ according to a standard formalism $[36,37,43]$. This formalism yields the following average over $\phi_{p}$ according to

$$
\frac{d \sigma_{\mathrm{tw}}}{d \Omega}=\int \frac{d \phi_{p}}{2 \pi} \frac{I\left(\theta_{p}, \phi_{p}\right)}{I_{\mathrm{tw}}} \frac{d \sigma_{\mathrm{pw}}\left(\theta_{p}, \phi_{p}\right)}{d \Omega},
$$

where $I\left(\theta_{p}, \phi_{p}\right) \propto\left|p_{i} \cdot p_{0}\left(\theta_{p}, \phi_{p}\right)\right|$ is the flux factor corresponding to a plane-wave component of the twisted photon interacting with the plane-wave electron, and $I_{\mathrm{tw}}$ is the socalled twisted flux factor, which can be defined [43] as the average of $I\left(\theta_{p}, \phi_{p}\right)$ over $\phi_{p}$. For scenarios where the problem possesses azimuthal symmetry, such as when the electron is at rest or when the motion of electron and photon are collinear, then the flux factors in Eq. (11) cancel. Finally, we note that for the scattering of a pure twisted Bessel particle, as described in Eq. (6), from a free plane-wave particle, $d \sigma_{\mathrm{tw}}$ is independent of $m$ [43] but still depends on the cone opening angle $\theta_{p}$.

\section{Differential cross section for a plane-wave component}

In this section, the formalism for the Compton scattering of a plane-wave photon from a plane-wave electron in uniform linear motion is adapted for an arbitrary plane-wave component of a twisted photon. This result is then used to evaluate the twisted cross section defined in Eq. (11).

The $S$ matrix for Compton scattering is determined according to the Feynman rules of QED to lowest order by [44]

$$
S_{\mathrm{pw}}=i(2 \pi \hbar)^{4} \delta^{(4)}\left(p_{i}+p_{0}-p_{f}-p_{1}\right) \mathcal{M}_{\mathrm{pw}},
$$

where the subscript pw denotes the plane-wave formalism, $p_{i}$ is the 4-momentum of the initial electron, $p_{0}$ is the 4momentum of the incident photon, $p_{1}$ is the 4-momentum of the scattered photon, while $p_{f}$ is the 4-momentum of the scattered electron, and the $\delta$ function ensures the conservation of the 4-momenta for the scattering process. The scattering amplitude $\mathcal{M}_{\mathrm{pw}}$ is given by

$$
\mathcal{M}_{\mathrm{pw}}=\frac{m_{e} c^{2} e^{2} \hbar}{2 \epsilon_{0} V^{2} \sqrt{E_{i} E_{f} E_{0} E_{1}}} \mathcal{K}_{\mathrm{pw}},
$$

where $V$ is the quantization volume, $E_{0}$ is the total energy of the incident photon, $E_{i}$ is the total energy of the initial electron, $E_{1}$ is the energy of the scattered photon, and $E_{f}$ the energy of the scattered electron. The associated amplitude $\mathcal{K}_{\mathrm{pw}}=\mathcal{K}_{1}+\mathcal{K}_{2}$, where

$$
\begin{aligned}
& \mathcal{K}_{1}=\bar{u}_{\lambda^{\prime}}\left(p_{f}\right) \hat{\epsilon}^{\alpha_{1}^{*}} \mathcal{G}\left(p_{i}+p_{0}\right) \hat{\epsilon}^{\alpha_{0}} u_{\lambda}\left(p_{i}\right), \\
& \mathcal{K}_{2}=\bar{u}_{\lambda^{\prime}}\left(p_{f}\right) \hat{\epsilon}^{\alpha_{0}} \mathcal{G}\left(p_{i}-p_{1}\right) \hat{\epsilon}^{\alpha_{1}^{*}} u_{\lambda}\left(p_{i}\right),
\end{aligned}
$$

while $\mathcal{G}=G / i \hbar$, and $G$ is the electron propagator. The quantity $u_{\lambda}(p)$ denotes the Dirac bispinor for an electron of definite helicity $\lambda$. The Dirac bispinors are normalized in this paper according to the convention $\bar{u}_{\lambda} u_{\lambda}=1$, and $\bar{u}_{\lambda}=u_{\lambda}^{\dagger} \gamma^{0}$ is the Dirac adjoint.

The calculation of the polarization-resolved, angledifferential cross section, in which the electron is initially unpolarized and the final electron is not measured, follows from (12)-(14) according to the standard treatment [44,45]. However, when the electron is also moving in the laboratory frame, an alternative procedure is often utilized, the so-called frame-hopping technique, to evaluate the cross section for Compton scattering [46]. That is, the problem is transformed (i.e., a Lorentz transformation is performed) to the rest frame of the electron, the well-known differential cross section for Compton scattering from electrons at rest is evaluated, and finally the problem is transformed back to the laboratory frame. We emphasize, however, that all final results are calculated in the laboratory frame. It is only the final result of this procedure, for each plane-wave component, evaluated in the laboratory frame, which is substituted into Eq. (11) to determine the twisted cross section.

Here we adapt the results of Ref. [46] to evaluate the differential cross section corresponding to a plane-wave component of the twisted photon scattering from a plane-wave electron. Translating their expressions into the symbols used in this paper, the result is

$$
\begin{aligned}
& \left(\frac{d \sigma_{\mathrm{pw}}\left(\theta_{p}, \phi_{p}\right)}{d \Omega}\right)_{\alpha_{0}, \alpha_{1}} \\
& \quad=\mathcal{F}_{0}\left[\mathcal{F}_{1} Q_{1}\left(\epsilon^{\alpha_{0}}\left(\phi_{p}\right), \epsilon^{\alpha_{1}}\right)+Q_{2}\left(\epsilon^{\alpha_{0}}\left(\phi_{p}\right), \epsilon^{\alpha_{1}}\right)\right],
\end{aligned}
$$

where

$$
\mathcal{F}_{0}=\frac{r_{e}^{2}}{4 \gamma_{i}^{2}\left(1-\vec{\beta}_{i} \cdot \vec{n}_{0}\left(\phi_{p}\right)\right)^{2}}\left(\frac{E_{1}\left(\phi_{p}\right)}{E_{0}}\right)^{2}
$$

and

$$
\mathcal{F}_{1}=\frac{E_{1}\left(\phi_{p}\right)\left(1-\vec{\beta}_{i} \cdot \vec{n}_{1}\right)}{E_{0}\left(1-\vec{\beta}_{i} \cdot \vec{n}_{0}\left(\phi_{p}\right)\right)}+\frac{E_{0}\left(1-\vec{\beta}_{i} \cdot \vec{n}_{0}\left(\phi_{p}\right)\right)}{E_{1}\left(\phi_{p}\right)\left(1-\vec{\beta}_{i} \cdot \vec{n}_{1}\right)},
$$


while

$$
Q_{1}\left(\epsilon^{\alpha_{0}}, \epsilon^{\alpha_{1}}\right)=\left|\mathcal{P}\left(\epsilon^{\alpha_{0}}, \epsilon^{\alpha_{1}^{*}}\right)\right|^{2}-\left|\mathcal{P}\left(\epsilon^{\alpha_{0}}, \epsilon^{\alpha_{1}}\right)\right|^{2}+1
$$

and

$$
Q_{2}\left(\epsilon^{\alpha_{0}}, \epsilon^{\alpha_{1}}\right)=2\left(\left|\mathcal{P}\left(\epsilon^{\alpha_{0}}, \epsilon^{\alpha_{1}^{*}}\right)\right|^{2}+\left|\mathcal{P}\left(\epsilon^{\alpha_{0}}, \epsilon^{\alpha_{1}}\right)\right|^{2}-1\right) .
$$

In $\mathcal{F}_{0}$, defined in Eq. (16), the classical electron radius is $r_{e}=$ $\alpha \hbar / m_{e} c \approx 2.82 \mathrm{fm}$, where $\alpha \approx 1 / 137036$ is the fine structure constant, the dimensionless velocity of the initial electron is $\vec{\beta}_{i}=\vec{p}_{i} / \gamma_{i} m_{e} c$, and the Lorentz factor $\gamma_{i}=E_{i} / m_{e} c^{2}$, whereas $E_{0}$ is the energy of the incident twisted photon, and the energy of the scattered photon is $E_{1}\left(\phi_{p}\right)$. This energy depends on $\phi_{p}$ as

$$
E_{1}\left(\phi_{p}\right)=\frac{E_{0}\left(1-\vec{\beta}_{i} \cdot \vec{n}_{0}\left(\phi_{p}\right)\right)}{\left(1-\vec{\beta}_{i} \cdot \vec{n}_{1}\right)+r_{0}\left(1-\cos \chi\left(\phi_{p}\right)\right)},
$$

where $\vec{n}_{0}$ and $\vec{n}_{1}$ are the unit vectors defined in Eq. (1), the generalized recoil parameter is given by

$$
r_{0}=\frac{E_{0}}{\gamma_{i} m_{e} c^{2}},
$$

and the component scattering angle $\chi\left(\phi_{p}\right)$ is defined by

$$
\cos \chi\left(\phi_{p}\right)=\vec{n}_{1} \cdot \vec{n}_{0}\left(\phi_{p}\right) .
$$

The quantities $Q_{1}\left(\epsilon^{\alpha_{0}}, \epsilon^{\alpha_{1}}\right)$ and $Q_{2}\left(\epsilon^{\alpha_{0}}, \epsilon^{\alpha_{1}}\right)$, defined in Eqs. (18) and (19), respectively, are given in terms of a function $\mathcal{P}\left(\epsilon^{\alpha}, \epsilon^{\alpha^{\prime}}\right)$, which is defined for arbitrary polarization 4-vectors $\epsilon^{\alpha}$ and $\epsilon^{\alpha^{\prime}}$ according to [46]

$$
\begin{aligned}
\mathcal{P}\left(\epsilon^{\alpha}, \epsilon^{\alpha^{\prime}}\right)= & \epsilon^{\alpha} \cdot \epsilon^{\alpha^{\prime}}-\frac{\left(p_{i} \cdot \epsilon^{\alpha}\right)\left(p_{0} \cdot \epsilon^{\alpha^{\prime}}\right)}{p_{i} \cdot p_{0}}-\frac{\left(p_{i} \cdot \epsilon^{\alpha^{\prime}}\right)\left(p_{1} \cdot \epsilon^{\alpha}\right)}{p_{i} \cdot p_{1}} \\
& +\frac{\left(p_{i} \cdot \epsilon^{\alpha}\right)\left(p_{i} \cdot \epsilon^{\alpha^{\prime}}\right)\left(p_{0} \cdot p_{1}\right)}{\left(p_{i} \cdot p_{0}\right)\left(p_{i} \cdot p_{1}\right)} .
\end{aligned}
$$

Finally, averaging Eq. (15) over initial photon polarizations and summing over the scattered photon polarizations yields the sum-averaged cross section corresponding to a plane-wave component of the twisted photon

$$
\frac{d \sigma_{\mathrm{pw}}\left(\theta_{p}, \phi_{p}\right)}{d \Omega}=2 \mathcal{F}_{0}\left(\mathcal{F}_{1}-\mathcal{F}_{2}\right),
$$

where $\mathcal{F}_{0}$ is given by Eq. (16), $\mathcal{F}_{1}$ is given by Eq. (17), and $\mathcal{F}_{2}$ is given by

$$
\mathcal{F}_{2}=\frac{\sin ^{2} \chi\left(\phi_{p}\right)-\mathcal{B}_{1}+\mathcal{B}_{2}}{\left[\gamma_{i}\left(1-\vec{\beta}_{i} \cdot \vec{n}_{1}\right)\right]^{2}}
$$

where

$$
\mathcal{B}_{1}=\frac{2\left(1-\cos \chi\left(\phi_{p}\right)\right)\left(\vec{\beta}_{i} \cdot \vec{n}_{1}-\vec{\beta}_{i} \cdot \vec{n}_{0}\left(\phi_{p}\right) \cos \chi\left(\phi_{p}\right)\right)}{1-\vec{\beta}_{i} \cdot \vec{n}_{0}\left(\phi_{p}\right)}
$$

and

$$
\mathcal{B}_{2}=\frac{\left(1-\cos \chi\left(\phi_{p}\right)\right)^{2}\left(\left|\vec{\beta}_{i}\right|^{2}-\left(\vec{\beta}_{i} \cdot \vec{n}_{0}\left(\phi_{p}\right)\right)^{2}\right)}{\left[1-\vec{\beta}_{i} \cdot \vec{n}_{0}\left(\phi_{p}\right)\right]^{2}} .
$$

The sum-averaged angle-differential cross section in Eq. (24) is substituted into Eq. (11) to evaluate the associated sum-averaged twisted cross section, and the polarizationresolved cross sections, given by Eq. (15), are likewise used to evaluate Eq. (11) to obtain the associated polarizationresolved twisted cross sections. The latter are required to determine the Stokes parameters of the scattered photons.

\section{Stokes parameters}

The polarization characteristics of the light scattered from the electron can be described with Stokes parameters. The version of the Stokes parameters adopted here utilizes the quantities, $P_{1}, P_{2}, P_{3}$ consistent with Refs. [24,25]. Here $P_{1}$ describes the degree to which the scattered light is linearly polarized in the scattering plane versus being linearly polarized perpendicular to it. It is defined by

$$
P_{1}=\frac{\left(\frac{d \sigma}{d \Omega}\right)^{1}-\left(\frac{d \sigma}{d \Omega}\right)^{2}}{\left(\frac{d \sigma}{d \Omega}\right)^{1}+\left(\frac{d \sigma}{d \Omega}\right)^{2}},
$$

where the superscripts of $\left(\frac{d \sigma}{d \Omega}\right)^{1}$ and $\left(\frac{d \sigma}{d \Omega}\right)^{2}$ correspond to $\alpha_{1}=1$ and $\alpha_{1}=2$, respectively, of the first pair of linear polarization 4-vectors, defined in Eq. (3), for the scattered photons. Likewise the Stokes parameter $P_{2}$ describes a degree of linear polarization according to

$$
P_{2}=\frac{\left(\frac{d \sigma}{d \Omega}\right)^{+}-\left(\frac{d \sigma}{d \Omega}\right)^{-}}{\left(\frac{d \sigma}{d \Omega}\right)^{+}+\left(\frac{d \sigma}{d \Omega}\right)^{-}},
$$

where the superscripts of $\left(\frac{d \sigma}{d \Omega}\right)^{+}$and $\left(\frac{d \sigma}{d \Omega}\right)^{-}$refer to the corresponding polarizations as defined in Eq. (4) for the second pair of linear polarization 4-vectors. Next, the degree of circular polarization of the scattered light is characterized by Stokes parameter $P_{3}$ according to

$$
P_{3}=\frac{\left(\frac{d \sigma}{d \Omega}\right)^{+1}-\left(\frac{d \sigma}{d \Omega}\right)^{-1}}{\left(\frac{d \sigma}{d \Omega}\right)^{+1}+\left(\frac{d \sigma}{d \Omega}\right)^{-1}},
$$

where the superscripts of the cross sections $\left(\frac{d \sigma}{d \Omega}\right)^{+1}$ and $\left(\frac{d \sigma}{d \Omega}\right)^{-1}$ correspond to scattered photons of positive and negative helicity, respectively, as defined in Eq. (5). Finally, the degree of polarization of the scattered light is then determined by

$$
\Pi=\sqrt{P_{1}^{2}+P_{2}^{2}+P_{3}^{2}} .
$$

The Stokes parameters as given by Eqs. (28)-(30) accommodate the preparation of an arbitrary initial state for the photon and for electron. Therefore they apply equally to incident plane-wave or twisted photons provided that the appropriate cross sections for each case are substituted. Throughout the discussion of the results concerning the Stokes parameters in the following section, it is assumed that the initial electron is unpolarized, the final electron is not measured, and the incident photon, whether plane-wave or twisted, is of definite helicity.

\section{RESULTS AND DISCUSSION}

\section{A. Angular distributions for motion along a common axis}

First, we consider the two cases in which the angular distributions possess azimuthal symmetry. This occurs if the twisted photon and plane-wave electron move in opposite 

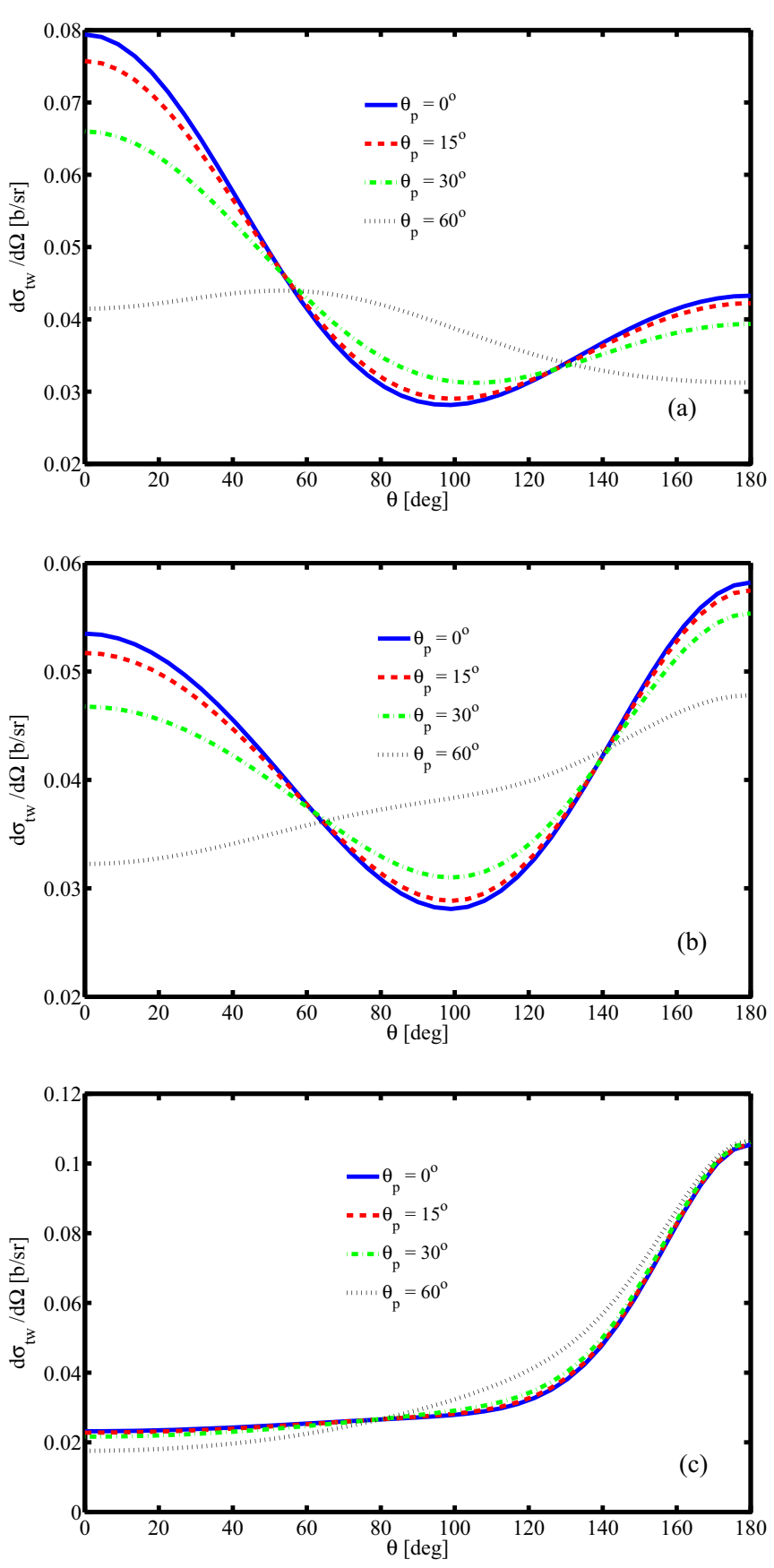

FIG. 2. Differential cross section $d \sigma_{\mathrm{tw}} / d \Omega$ for Compton scattering of a photon prepared in a twisted Bessel state, assumed unpolarized, as a function of the scattering angle $\theta$ for several values of the cone opening angle $\theta_{p}$. The collision is head on (i.e., $\theta_{i}=180^{\circ}$ ) with a plane-wave electron. The energy of the incident photon is $E_{0}=100 \mathrm{keV}$ while the kinetic energy of the electron is (a) $T_{i}=0$, (b) 10 , and (c) $100 \mathrm{keV}$. The case $\theta_{p}=0^{\circ}$ corresponds to a plane-wave photon.

directions, a so-called head-on (i.e., $\theta_{i}=180^{\circ}$ ) collision, or they collide while moving in the same direction (i.e., $\theta_{i}=0^{\circ}$ ), which shall be referred to henceforth as a same-direction collision.

The sum-averaged twisted differential cross sections for a head-on (i.e., $\theta_{i}=180^{\circ}$ ) collision are presented in Fig. 2 as a function of the scattering angle $\theta$ and for several values of the cone opening angle $\theta_{p}$ of the twisted photon. The results are shown for three choices of the initial electron kinetic energy $T_{i}$. Namely, $T_{i}=0 \mathrm{keV}$ in Fig. $2(\mathrm{a}), T_{i}=10 \mathrm{keV}$ in Fig. 2(b), and finally $T_{i}=100 \mathrm{keV}$ in Fig. 2(c). The energy of the incident photon is $E_{0}=100 \mathrm{keV}$ and is considered unpolarized.

As shown in Fig. 2(a) for the case where the electron is at rest in the laboratory frame, the effect of increasing the opening angle $\theta_{p}>0^{\circ}$ is to decrease the amount of scattering about the forward and backward directions and increase the amount of scattering for a more intermediate range of $\theta$. This is in agreement with results reported previously [24,25]. However, as the kinetic energy of the electron increases, shown in Figs. 2(b) and 2(c), in addition to the expected shift of the distributions toward greater backscattering, the angular distributions become progressively less sensitive to the opening angle $\theta_{p}$ of the twisted photon; that is, the results for the scattering of a twisted photon differ progressively less from the results for the scattering of a plane-wave photon as the speed of the electron increases.

In Fig. 3, the sum-averaged cross sections are presented as a function of the scattering angle $\theta$ for several values of the cone opening angle $\theta_{p}$. The organization of the figure is the same as for Fig. 2 except, for the electron, $\theta_{i}=0^{\circ}$. As can be seen from the figure, in contrast to the head-on collision, as $T_{i}$ increases, the dependence of the angular distributions on the opening angle $\theta_{p}$ is enhanced, at least in the forward scattering direction; that is, the results for the scattering of a twisted photon progressively differ from those for a planewave photon as the speed of the electron increases.

Furthermore, in order to compare the response with lowenergy twisted photons, especially in the Thomson limit, the calculations corresponding to Figs. 2 and 3 were repeated with the same choices of the electron energy and the twisted photon opening angles, except that the energy of the photon was chosen to be $10 \mathrm{eV}$. For these distributions, which are not shown here, the greatest contrast with the distributions of the $100 \mathrm{keV}$ photon occurs when the electron is at rest. In that case, the low-energy distributions have the expected symmetry with respect to forward and backward scattering and vary with opening angle $\theta_{p}$ as already described in Ref. [24], whereas the distributions displayed in Fig. 2(a) are, as expected, asymmetric with respect to forward-backward scattering. As the energy of the electron increases, however, this contrast diminishes, until the energy of the incident twisted photon apparently plays a greatly reduced role in influencing the distributions. In the case of a $10 \mathrm{eV}$ twisted photon and an electron of $100 \mathrm{keV}$ in a head-on collision, except for possibly their absolute magnitudes, the angular distributions closely resemble the appearance of those in Fig. 2(c) and, therefore, are also nearly insensitive to the opening angle $\theta_{p}$ of the twisted photon. For a $10 \mathrm{eV}$ twisted photon and an electron of $100 \mathrm{keV}$ in a same-direction collision, the appearance of the angular distributions closely resembles those for Fig. 3(c). In this case, the angular distributions have become nearly insensitive to the twisted photon energy but not its opening angle.

Angular distributions of the Stokes parameters $P_{1}$ (left column) and $P_{3} / \Lambda_{0}$ (middle column), and degree of polarization 

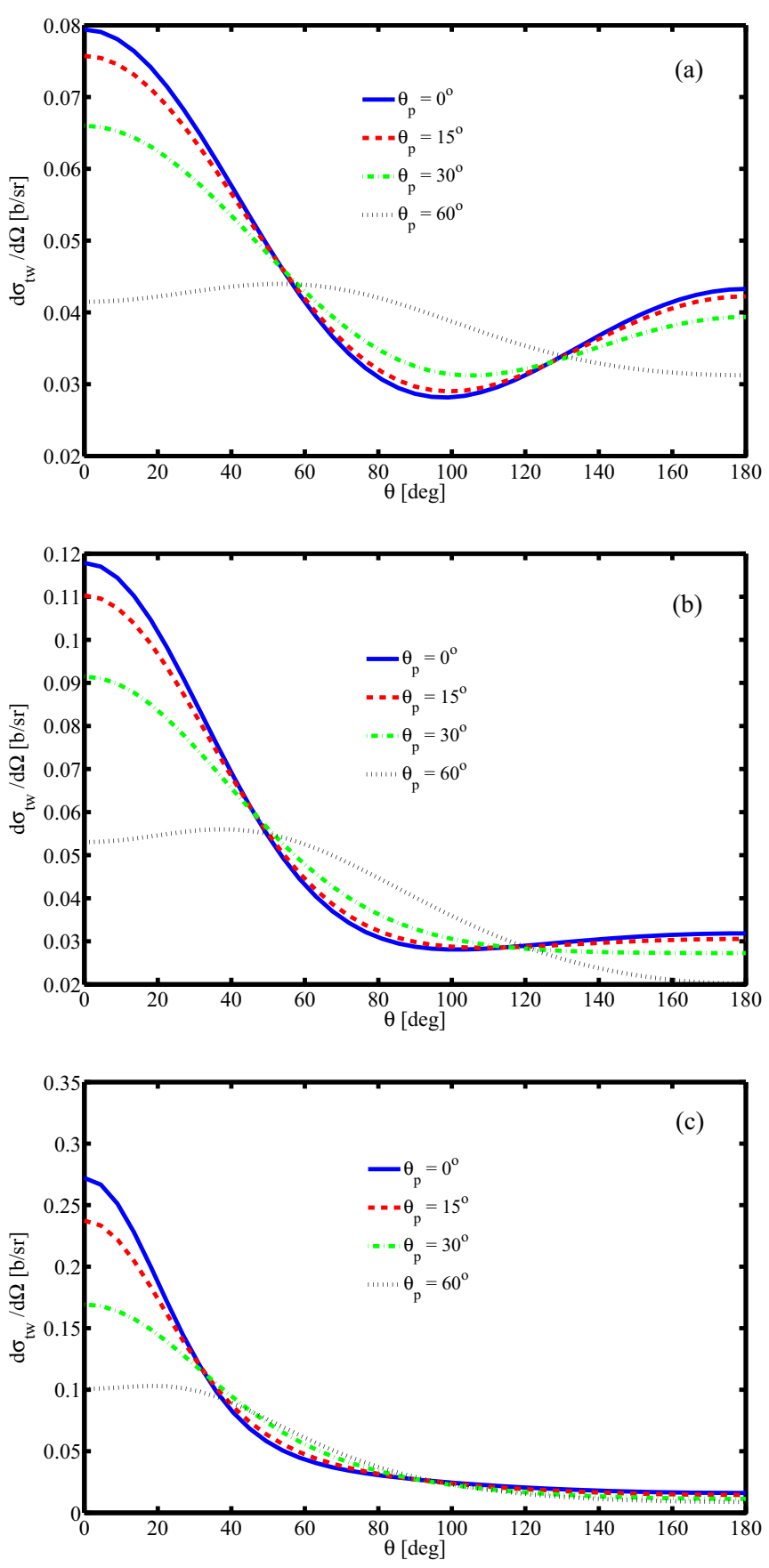

FIG. 3. Differential cross section $d \sigma_{\mathrm{tw}} / d \Omega$ for Compton scattering of a photon prepared in a twisted Bessel state, assumed unpolarized, as a function of the scattering angle $\theta$ for several values of the cone opening angle $\theta_{p}$. The collision is same-directional (i.e., $\theta_{i}=0^{\circ}$ ) with a plane-wave electron. All other parameters and the organization of the figure are the same as for Fig. 2.

$\Pi$ (right column) are shown in Fig. 4 for a head-on (i.e., $\theta_{i}=180^{\circ}$ ) collision and for several choices of opening angle $\theta_{p}$. The electron kinetic energy is $T_{i}=0$ (first row), 10 (second row), and $100 \mathrm{keV}$ (third row). The energy of the incident twisted photon is $E_{0}=100 \mathrm{keV}$ and is of definite helicity $\Lambda_{0}$. The Stokes parameter $P_{2}$ is omitted here as its value in this case is always negligibly close to zero.
The Stokes parameter $P_{1}$, corresponding to an electron at rest, is shown in the figure (first row, left column). As the opening angle $\theta_{p}>0^{\circ}$ increases, the minima become progressively less negative in comparison to the pronounced minimum displayed by the distribution for plane-wave scattering. As $T_{i}$ increases (second and third rows, left column), in addition to the expected shift of the minima toward larger scattering angles $\theta$, the sensitivity of the response to $\theta_{p}$ is reduced; that is, for identically prepared incident twisted photons scattering from electrons moving toward them at different speeds, the distribution of $P_{1}$ arising from an electron moving with greater kinetic energy (higher speed) more closely resembles the distribution for scattering of the plane-wave photon than from an electron moving with less kinetic energy (lower speed).

The distributions for $P_{3} / \Lambda_{0}$ in the figure (middle column) follow trends similar to those for $P_{1}$. Considering first the scattering from an electron at rest (first row, middle column), an increase of the opening angle $\theta_{p}$ tends to reduce the magnitude of $P_{3} / \Lambda_{0}$ over most values of $\theta$. Moreover, as $T_{i}$ increases (second and third rows, middle column), the sensitivity of this response to $\theta_{p}$ is reduced.

Finally, the distributions for the degree of polarization $\Pi$ in the figure (right column) reflect the drop in polarization with increasing opening angle exhibited by $P_{1}$ and $P_{3}$. Like them, increasing $T_{i}$ reduces the response of $\Pi$ to changes of $\theta_{p}$.

The Stokes parameters $P_{1}, P_{3} / \Lambda_{0}$, and the partial polarization $\Pi$ as a function of the scattering angle $\theta$ are displayed in Fig. 5. The organization of the figure and all other parameters are identical to those for Fig. 4 except here the electron is moving in the same direction (i.e., $\theta_{i}=0^{\circ}$ ) as the incident photon. In this case, as shown in the figure, as the electron speeds up (i.e., $T_{i}$ increases) the distributions become increasingly sensitive to $\theta_{p}>0^{\circ}$, opposite to the trend for a head-on collision.

Moreover, similar to the aforementioned angular distributions of the sum-averaged cross sections, further calculations with an incident twisted photon of much low energy $(10 \mathrm{eV})$ indicate that, as the electron becomes increasingly energetic, the angular distributions of the Stokes parameters begin to resemble those for an incident photon of higher energy $(100 \mathrm{keV})$ under identical conditions. That is, increasing the electron energy diminishes the sensitivity of the angular distributions to the energy of the incident twisted photon, but the sensitivity to the opening angle is diminished only for the case of scattering head-on from the electron.

Parenthetically, in order to thoroughly explore the parameter space involved, the results presented in Figs. 2-5 examine the scattering response for a somewhat energetic $(100 \mathrm{keV})$ twisted photon for selected opening angles representative of the range $\theta_{p} \in\left[0^{\circ}, 60^{\circ}\right]$. In fact, the opening angles of twisted photons typically produced in experiments are small $\left(\lesssim 1^{\circ}\right)$, so that much of this range currently remains inaccessible. However, the field of x-ray optics is advancing rapidly [47,48], including the shaping of twisted beams [49,50], and the technical challenges to produce high-energy twisted photons with large $\left(\gg 1^{\circ}\right)$ opening angles are likely to be overcome in the future. Ultimately, however, the use of small opening angles in the calculations do not alter the main results of this paper. Nevertheless, we shall compare the results for 

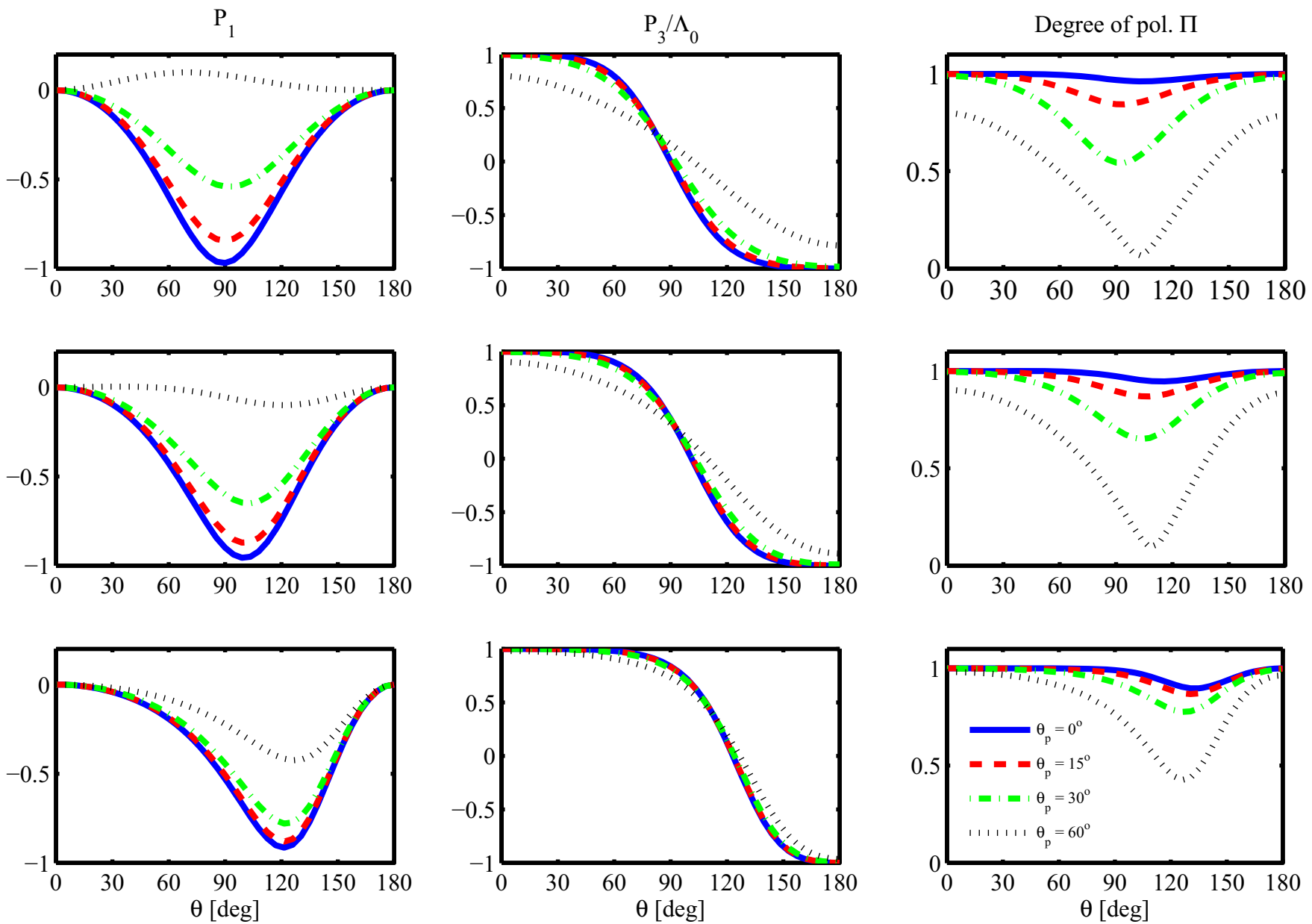

FIG. 4. Stokes parameters $P_{1}$ (first column), $P_{3} / \Lambda_{0}$ (second column), and the degree of polarization $\Pi$ (third column) of the Comptonscattered photons as a function of $\theta$ for several choices of momentum-cone opening angle $\theta_{p}$. The twisted photon has well-defined helicity $\Lambda_{0}$ and energy $E_{0}=100 \mathrm{keV}$. The collision is head-on (i.e., $\theta_{i}=180^{\circ}$ ). First row: $T_{i}=0 \mathrm{keV}$, second row: $T_{i}=10 \mathrm{keV}$, and third row: $T_{i}=100 \mathrm{keV}$. The common legend for all panels in the figure is shown in the lower right panel.

small and large opening angles at several points later in the discussion.

As a final note, none of the results presented so far display an explicit dependence on the intrinsic orbital angular momentum carried by the incident photon. Indeed, the same results are obtained even if $m=\Lambda= \pm 1$, which arises only from the helicities of the plane-wave components of the Bessel state. This independence was briefly alluded to at the end of Sec. II B. It is the expected result [43] whenever a particle that is well approximated by a pure twisted Bessel state, as in Eq. (6), scatters from another particle that is well approximated by a plane wave.

\section{B. Critical velocity}

The results presented in Figs. 2-5 indicate that the electron motion influences the dependence of the various distributions on the opening angle $\theta_{p}$ of the twisted photon. In order to shed some light on this influence, it is useful to visualize the process in the rest frame of the electron. However, we stress once more that the calculation of all distributions in this paper are, in fact, performed in the laboratory frame.
In this section, the electron has $\beta_{x}=\beta_{y}=0$ and $\beta_{z}=$ $\pm\left|\vec{\beta}_{i}\right|$ in the laboratory frame. In this case, the plane-wave components of the twisted photon will still sweep out a cone when viewed in the electron rest frame, but each component will be oriented at a generally different cone opening angle $\theta_{p}^{\prime}$ to the common $z z^{\prime}$ axis, where the prime indicates a quantity in the rest frame. Performing the Lorentz transformation yields

$$
\cos \theta_{p}^{\prime}=\frac{\cos \theta_{p}-\beta_{z}}{1-\beta_{z} \cos \theta_{p}} .
$$

According to Eq. (32), if the electron is involved in a headon collision, then $\beta_{z}=-\left|\vec{\beta}_{i}\right|$, and the greater the value of $\beta_{z}$, the smaller the opening angle of the twisted photon will be in the electron rest frame. For example, for an electron traveling with $T_{i}=100 \mathrm{keV}$ in the negative $z$ direction $\left(\beta_{z} \approx-0.55\right)$ and colliding head-on with a twisted photon traveling in the opposite direction in the laboratory frame with an opening angle of $\theta_{p}=30^{\circ}$, the opening angle of the twisted photon will be only $\theta_{p}^{\prime} \approx 16.5^{\circ}$ in the rest frame of the electron. Thus, in this case, the twisted photon behaves substantially more like a plane-wave photon, and this increased similarity will be 

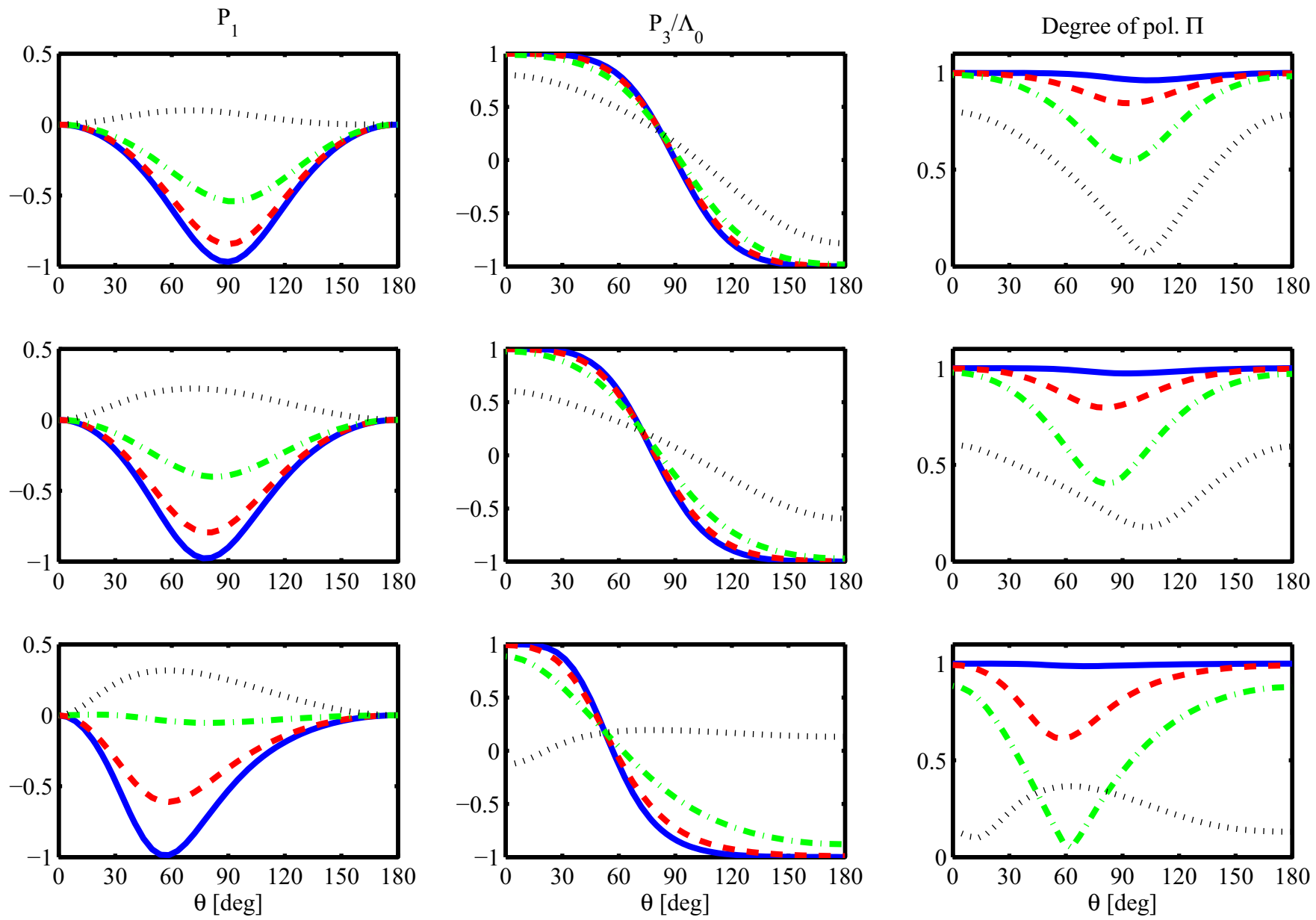

FIG. 5. Stokes parameters $P_{1}, P_{3} / \Lambda_{0}$, and the degree of polarization $\Pi$ of the Compton scattered photons as a function of $\theta$ for several choices of opening angle $\theta_{p}$. The organization of the figure is the same as for Fig. 4, except $\theta_{i}=0^{\circ}$. The legend for all panels in the figure is also the same as for Fig. 4.

reflected by the various angular distributions in both the rest frame of the electron and also back in the laboratory frame.

If an electron is involved in a same-direction collision (i.e., $\theta_{i}=0^{\circ}$ ) with the twisted photon, which is propagating in the positive $z$ direction, then $\beta_{z}=\left|\vec{\beta}_{i}\right|$. According to Eq. (32), the opening angle of the twisted photon is now larger in the rest frame of the electron than in the laboratory frame.

For example, if an electron travels with $T_{i}=100 \mathrm{keV}$ $\left(\beta_{z} \approx 0.55\right)$ in the positive $z$ direction, a twisted photon with an opening angle of $\theta_{p}=30^{\circ}$ in the laboratory frame has an opening angle of $\theta_{p}^{\prime} \approx 52.7^{\circ}$ in the electron rest frame. This also enhances the effect of the opening angle on the angular distributions in the laboratory frame.

An interesting additional feature is revealed in the third row, middle column of Fig. 5 , which presents $P_{3} / \Lambda_{0}$ for a twisted photon in a same-direction collision with an electron of $T_{i}=100 \mathrm{keV}\left(\beta_{z} \approx 0.55\right)$. Interestingly, as the opening angle increases to $\theta_{p}=60^{\circ}$ in the laboratory frame, the distribution shifts sufficiently that it now becomes slightly negative for small $\theta$ and slightly positive for large $\theta$. That is, the forward-scattered photons are now beginning to manifest the opposite helicity to the incident photon and the backscattered photons are beginning to manifest the same helicity as the incident photon. This surprising feature does not occur for the scattering of a plane-wave photon under identical circumstances nor for a twisted photon scattering from an electron at rest.

According to Eq. (32), if $\theta_{p}=60^{\circ}$ and $T_{i}=100 \mathrm{keV}\left(\beta_{z} \approx\right.$ $0.55)$, then $\theta_{p}^{\prime} \approx 93.8^{\circ}$ in the electron rest frame. This result entails that the cone swept out by the plane-wave components of the twisted photon in the rest frame of the electron has become slightly inverted $\cos \theta_{p}^{\prime}<0$; alternately, the momentum cone can be thought of as opening up with acute angle about the negative $z$ direction.

It is possible to interpret these results in configuration space in terms of the twisted Bessel photon propagating more slowly along the $z$ direction than the electron as observed in the laboratory frame. Theoretically, this propagation velocity is often taken to be $c \cos \theta_{p}$, or the projection of the velocity of a plane-wave component along the average direction of motion, at least for a photon prepared in a pure twisted Bessel state in free space. For the aforementioned scenario with inversion, it could then be interpreted to reverse direction in the electron rest frame. This interpretation is consistent with the results reported in a recent experiment [51] using Hong-OuMandel interferometry to determine the rate of propagation of 
several classes of structured photons, including one prepared in a Bessel state, relative to a plane-wave photon. The results of that experiment indicated that a Bessel photon moves more slowly than the usual plane-wave photon. However, it is pointed out in [52] that there are many subtleties that must be considered when determining the velocity, or more precisely group velocity, of a structured photon, including alternate definitions for its computation. This problem becomes especially acute for models of realistic beams and pulses that attempt to take account of their finite temporal and spatial extent.

In this paper, we focus instead on interpreting the results in terms of the cone of plane-wave components in the momentum-space representation of the twisted photon. Based on Eq. (32), we define a critical velocity for an electron traveling in the positive $z$ direction (i.e., $\beta_{x}=\beta_{y}=0, \beta_{z}>$ 0 ), which indicates the onset of momentum-cone inversion, by imposing the condition $\cos \theta_{p}^{\prime}=0$. Solving this equation implies

$$
\beta_{z}^{c}=\cos \theta_{p},
$$

where $\beta_{z}^{c}$ designates the critical value of $\beta_{z}$. This critical velocity can be realized here only for $\beta_{z}>0$. When $\beta_{z}>\beta_{z}^{c}$, the momentum cone of the twisted photon inverts $\left(\cos \theta_{p}^{\prime}<0\right)$ in the electron rest frame. In this frame, the direction of photons backscattered relative to the direction of a component of the momentum cone begin to face into the positive $z^{\prime}$ direction and for those forward scattered, into the negative $z^{\prime}$ direction. Upon transformation back to the laboratory frame, the so-called backscattered photons in the elctron rest frame will tend to be focused into a smaller solid angle around the positive $z$ direction in the laboratory frame (headlight effect), and the so-called forward scattered photons spread into a larger solid angle about the negative $z$ direction. Hence, this analysis indicates that when $\beta_{z}>\beta_{z}^{c}$ the photon scattered into or near the positive $z$ direction, in the laboratory frame, should tend to have helicity (as measured by $P_{3}$ ) opposite to that of the incident twisted photon, and when scattered into or near the negative $z$ direction to tend have the same helicity as the incident Bessel photon. Moreover, the greater the value of $\beta_{z}$, the more pronounced this change in tendency for the helicity, the smaller the solid angle of the forward scattered, opposite-helicity region, and the larger the solid angle of the backscattered, like-helicity region.

To test these ideas, additional calculations were performed, in the laboratory frame, for two different twisted Bessel photons. The first was chosen with an energy $100 \mathrm{keV}$, definite helicity $\Lambda_{0}$, and opening angle $\theta_{p}=30^{\circ}$, whereas the second was chosen with an energy $10 \mathrm{eV}$, definite helicity $\Lambda_{0}$, and opening angle $\theta_{p}=3^{\circ}$. In both cases the calculations were performed for several choices of the kinetic energy of the electron. In all cases, of course, $\beta_{z}>0$. For the twisted photon of opening angle $\theta_{p}=30^{\circ}$, Eq. (33) indicates an expected $\beta_{z}^{c} \approx 0.87$ and associated critical kinetic energy $T_{i}^{c} \approx 511$ $\mathrm{keV}$, which is close in value to the rest mass of the electron entirely by coincidence. For the other twisted photon of opening angle $\theta_{p}=3^{\circ}$, Eq. (33) indicates an expected $\beta_{z}^{c} \approx 0.998$ and associated critical kinetic energy $T_{i}^{c} \approx 9.25 \mathrm{MeV}$.

The angular distributions of $P_{3} / \Lambda_{0}$ for the $100 \mathrm{keV}$ twisted photon are presented in Fig. 6(a). When the electron is at rest in the laboratory frame, the results are quite similar to those
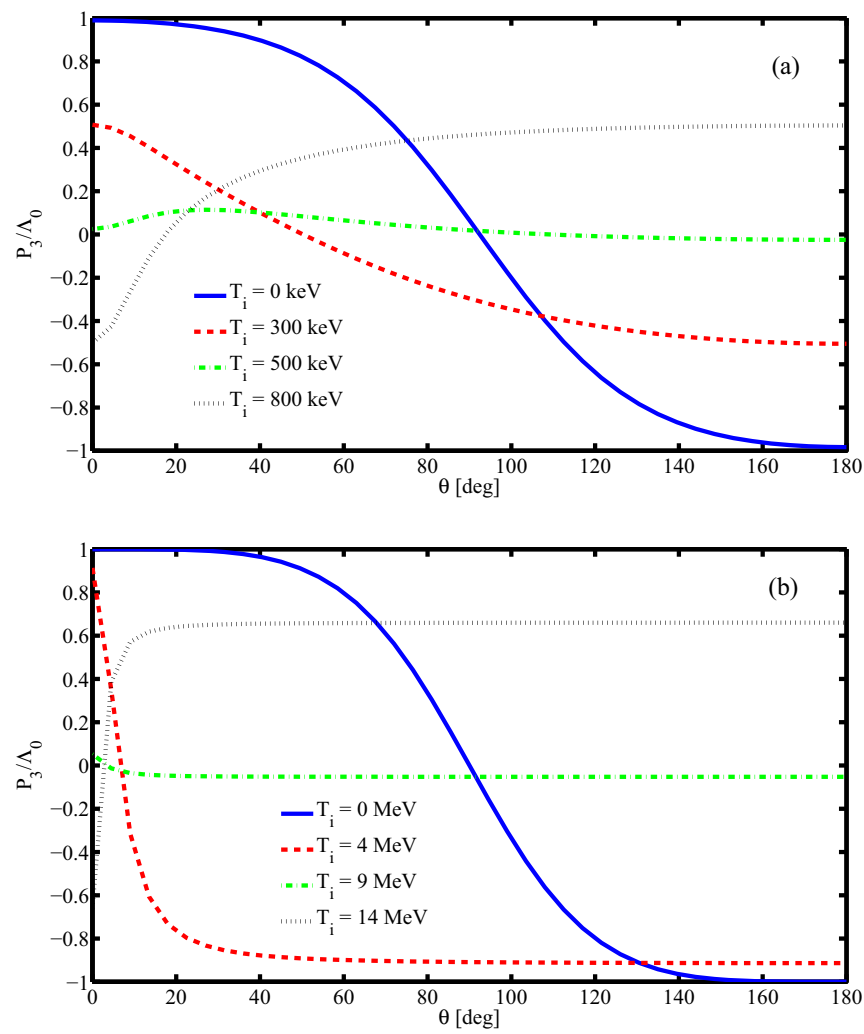

FIG. 6. Stokes parameter $P_{3} / \Lambda_{0}$ of the Compton-scattered photons as a function of $\theta$ for several choices of electron kinetic energy $T_{i}$ and $\theta_{i}=0^{\circ}$. The incident twisted photon in (a) has opening angle $\theta_{p}=30^{\circ}$ and energy $E_{0}=100 \mathrm{keV}$, whereas in (b) $\theta_{p}=3^{\circ}$ and $E_{0}=10 \mathrm{eV}$. In both cases, the photons have definite helicity $\Lambda_{0}$.

for a plane-wave photon even for the relatively large opening angle $\theta_{p}=30^{\circ}$. Most importantly, forward scattered photons have nearly the same helicity and backscattered photons have nearly opposite helicity to the incident twisted photon. However, as the kinetic energy of the electron increases, the distributions gradually change. For $T_{i}=300 \mathrm{keV}\left(\beta_{z} \approx 0.78\right)$, which is well below the critical value $\beta_{z}^{c}$ for this choice of $\theta_{p}$, the magnitude of $P_{3} / \Lambda_{0}$ has decreased over a wide range of scattering angles, but the helicities for forward-scattered photons and backscattered photons still correlate strongly with those from the electron-at-rest case and the plane-wave case. As the kinetic energy reaches $T_{i}=500 \mathrm{keV}\left(\beta_{z} \approx 0.86\right)$, a value only slightly less than the critical value, the quantity $P_{3} / \Lambda_{0}$ assumes values close to zero for nearly all scattering angles. Finally, as the electron reaches $T_{i}=800 \mathrm{keV}\left(\beta_{z} \approx\right.$ 0.92 ), which is much larger than the critical value, the $P_{3} / \Lambda_{0}$ is now of significant magnitude and of the opposite sign to its value for the electron-at-rest case and the plane-wave case over a wide range of $\theta$.

The angular distributions of $P_{3} / \Lambda_{0}$ are displayed in Fig. 6(b) for the $10 \mathrm{eV}$ twisted photon, a low-energy photon with a relatively small opening angle $\theta_{p}=3^{\circ}$. It is clear that the phenomenon involving the reversal of helicities of the scattered photons is not limited to a twisted photon of high energy or large cone opening angle. A twisted photon with a smaller opening angle simply requires a more energetic electron to induce the inversion of the momentum cone. 

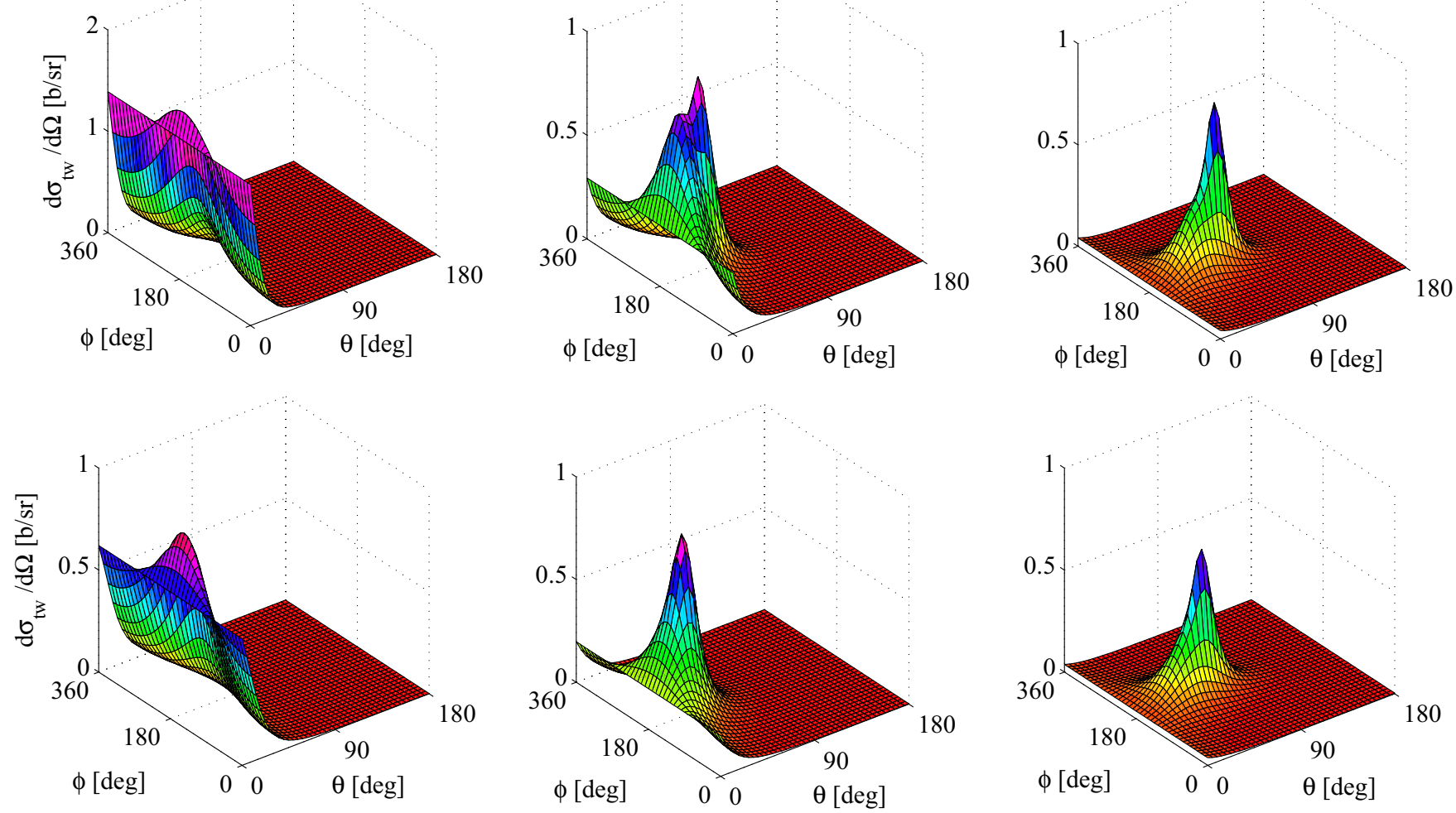

FIG. 7. Sum-averaged differential cross sections $d \sigma_{\mathrm{tw}} / d \Omega$ as a function of the scattering angles $\theta$ and $\phi$ for an incident plane-wave photon (top row) and twisted photon (bottom row) with opening angle $\theta_{p}=30^{\circ}$. The energy of the incident photon is $E_{0}=100 \mathrm{keV}$, whereas $T_{i}=$ $800 \mathrm{keV}$. Calculations were performed for several choices of the electron direction of motion specified by $\theta_{i}=10^{\circ}, \phi_{i}=180^{\circ}$ (first column), $\theta_{i}=30^{\circ}, \phi_{i}=180^{\circ}$ (second column), and $\theta_{i}=60^{\circ}, \phi_{i}=180^{\circ}$ (third column).

Moreover, the results of further calculations, not displayed here, on a $10 \mathrm{eV}$ twisted photon with opening angle $\theta_{p}=$ $30^{\circ}$ and the same choices for the electron kinetic energy yield angular distributions of $P_{3} / \Lambda_{0}$ nearly identical to those displayed in Fig. 6(a). Likewise, additional calculations on a $100 \mathrm{keV}$ twisted photon with opening angle $\theta_{p}=3^{\circ}$ and the same choices for the electron kinetic energy yield angular distributions of $P_{3} / \Lambda_{0}$ nearly identical to those displayed in Fig. 6(b).

These results indicate that the forward-scattered and backscattered photons arising from the twisted photon are now strongly correlated to the opposite helicity of those arising from a plane-wave photon or a twisted photon colliding with an electron at rest. The dependence of this reversal on cone opening angle $\theta_{p}$ and the insensitivity to the twisted photon energy are in agreement with the expectations of the preceding discussion connected with Eq. (33).

Finally, we note that strict forward scattering in the case of a same-direction collision between twisted photon and electron is not trivial, as it is for a plane-wave photon. That is, according to Fig. 3, strict forward scattering has the greatest likelihood to occur for plane-wave and twisted photon alike, but according to Eq. (20) the scattered photon has the same energy as the incident plane-wave photon regardless of the speed of the electron. On the other hand, for the twisted photon with $E_{0}=100 \mathrm{keV}$ and $\theta_{p}=30^{\circ}$ and the electron with $\beta_{z}=0.92$, from the previous discussion, Eq. (20) yields $E_{1} \approx 225 \mathrm{keV}$.

\section{Angular distributions for an electron moving in an arbitrary direction}

In this section, we examine the case whereby the electron can travel in an arbitrary direction relative to the motion of the twisted photon, which is still assumed to propagate along the positive $z$ direction. In particular, we explore the robustness of the results of the previous section, namely the sign reversal of $P_{3}$ for an electron whose speed exceeds a critical value, to this variation of the electron direction of motion.

Figure 7 illustrates the sum-averaged cross sections $d \sigma_{\mathrm{tw}} / d \Omega$ as a function of scattering angles $\theta$ and $\phi$ for an incident plane-wave photon (top row) and a twisted photon (bottom row) for several choices of the electron direction of motion, which are specified by $\theta_{i}$ and $\phi_{i}$. The energy of the incident photon (plane-wave or twisted) is $E_{0}=100 \mathrm{keV}$, and the opening angle of the twisted photon is $\theta_{p}=30^{\circ}$ whereas for the electron $T_{i}=800 \mathrm{keV}\left(\left|\vec{\beta}_{i}\right|=\beta_{i} \approx 0.92\right)$. This choice of $T_{i}$ was made in order to exceed the critical value for the choice of opening angle. Finally, $\phi_{i}=180^{\circ}$ throughout. As is clear from the figure, as $\theta_{i}$ increases, the angular distributions arising from plane-wave and twisted photons become increasingly similar. Further, the azimuthal symmetry of the distributions of the previous sections is clearly broken, but now the $x-z$ plane, which contains the momentum vector of the electron, defines the new symmetry.

Specifically, for polar angle $\theta_{i}=10^{\circ}$ (first column), the angular distribution of the cross section for the twisted photon 

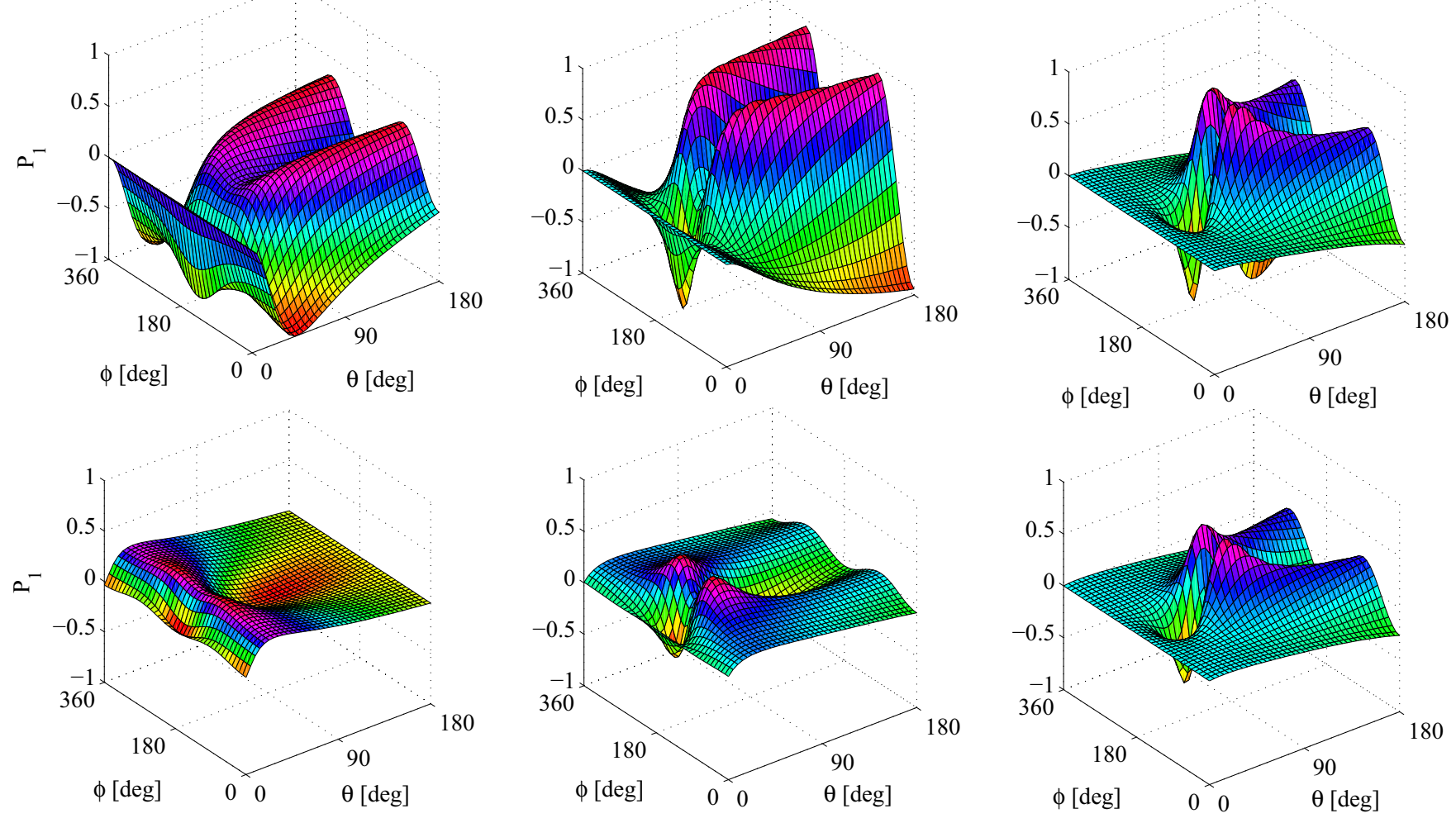

FIG. 8. Stokes parameter $P_{1}$ as a function of the scattering angles $\theta$ and $\phi$ for an incident plane-wave photon (top) and twisted photon (bottom) with opening angle $\theta_{p}=30^{\circ}$. The organization of the figure and all other parameters are the same as for Fig. 7, except the incident photon (plane-wave or twisted) has helicity $\Lambda_{0}=+1$.

(bottom panel) differs substantially from the corresponding plane-wave case (top panel). The difference is especially acute for the peak values of the distributions; the peak value for the twisted photon is less than half the peak value for the planewave photon. As $\theta_{i}$ increases the distributions change. For $\theta_{i}=60^{\circ}$ (third column), the plane-wave and twisted angular distributions are now quite similar in shape and the peak value for the twisted photon is only slightly smaller than for the plane-wave photon. For even larger values of $\theta_{i}$, further calculations, which are not displayed here, indicate that the angular distributions for the plane-wave and the twisted photon become even more similar.

We next consider the Stokes parameters. In Fig. 8, the Stokes parameter $P_{1}$ is plotted as a function of the scattering angles $\theta$ and $\phi$ for an incident plane-wave photon (top row) and twisted photon (bottom row) with opening angle $\theta_{p}=$ $30^{\circ}$. The organization of the figure and all other parameters are the same as for Fig. 7, except that here the twisted photon has definite helicity $\Lambda_{0}=+1$. For the choice of $\theta_{i}=10^{\circ}$ (first column) the distribution from the twisted photon is quite different from the distribution from a plane-wave photon. As $\theta_{i}$ continues to increase, the distributions change until $\theta_{i}=$ $60^{\circ}$ (third column) the distributions for plane-wave photon and twisted photon already closely resemble each other.

The corresponding angular distributions for the Stokes parameter $P_{2}$ are shown in Fig. 9. The organization of the figure and all other parameters are the same as for Fig. 8. Unlike the results in the previous section, $P_{2}$ now depends significantly on the scattering angles $\theta$ and $\phi$ for both an incident plane-wave photon (top row) and twisted photon (bottom row). Similar to the behavior of $P_{1}$, when $\theta_{i}$ is small, such as for $\theta_{i}=10^{\circ}$ (first column), the distribution from the twisted photon is quite different from the one for a plane-wave photon, but as $\theta_{i}$ continues to increase, the distributions of $P_{2}$ for a plane-wave and a twisted photon begin to resemble each other.

The Stokes parameter $P_{3}$ as a function of $\theta$ and $\phi$ is shown in Fig. 10 for several choices of $\theta_{i}$. The organization of the figure and all other parameters are the same as for Fig. 8 . As noted previously, for a twisted photon of definite helicity scattering from an electron traveling in the same direction (here the $z$ direction), there is a continuous but well-defined transition in which $P_{3}$ undergoes a sign shift relative to that from the scattering of a plane-wave photon over a wide range of $\theta$, the difference of which is most prominent about the strictly forward direction $\left(\theta \rightarrow 0^{\circ}\right)$ and about the backward direction $\left(\theta \rightarrow 180^{\circ}\right)$. This sign shift occurs if $\beta_{z}>\beta_{z}^{c}$, which correlates with the momentum cone inversion of the twisted photon in the rest frame of the electron.

The results for $\theta_{i}=10^{\circ}$ (first column) in Fig. 10 indicate that this relative sign shift persists even when the direction of the electron motion is not perfectly aligned with the direction of the twisted photon motion; it is robust to small variations in $\theta_{i}$ and not simply an artifact of the special arrangement of perfect alignment (i.e., here $\theta_{i}=0^{\circ}$ ).

However, with an increase to $\theta_{i}=30^{\circ}$ (second column), the angular distributions of $P_{3}$ from twisted and plane-wave photons are beginning to resemble each other. Moreover, the 

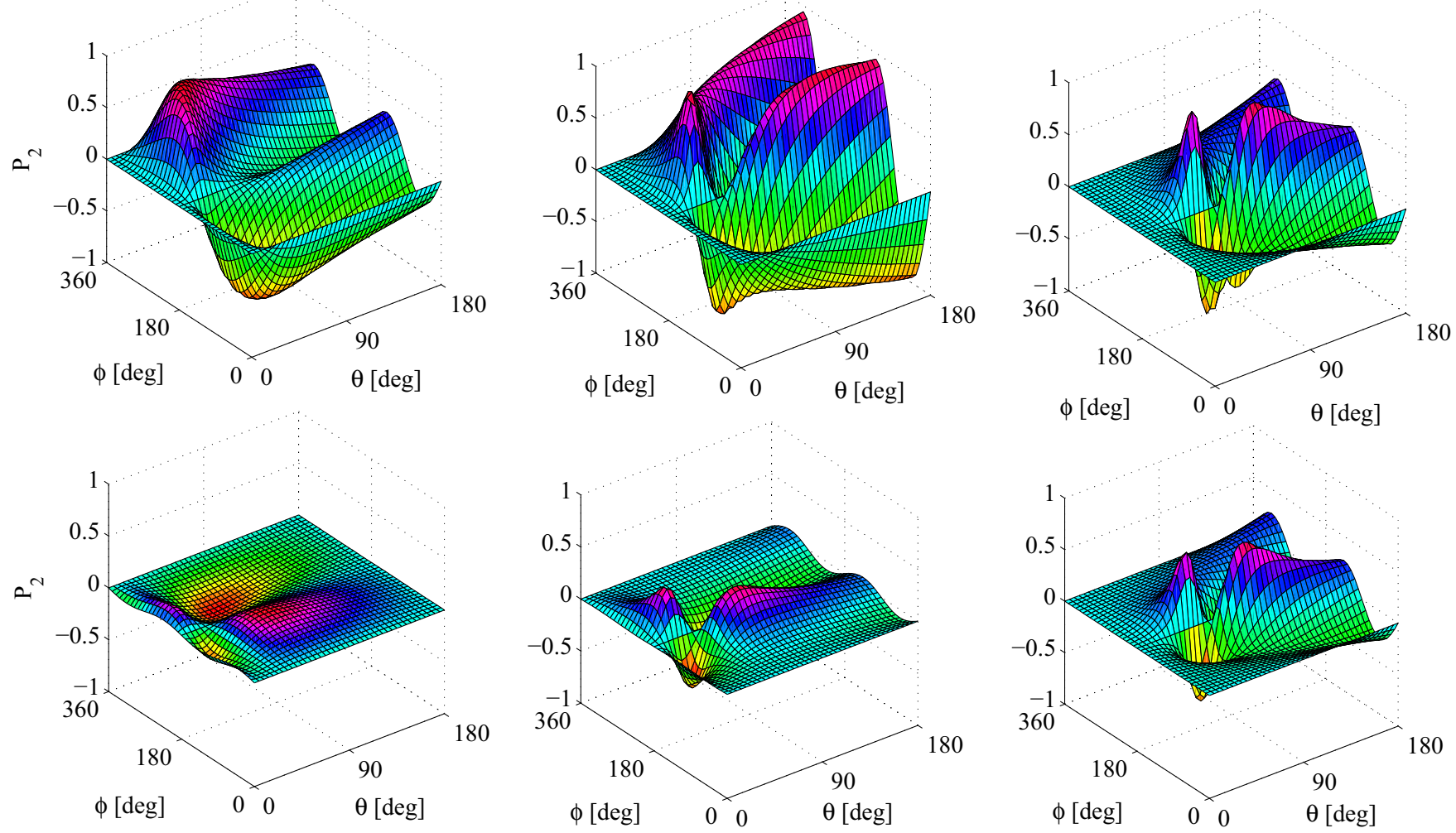

FIG. 9. Stokes parameter $P_{2}$ as a function of the scattering angles $\theta$ and $\phi$ for an incident plane-wave photon (top) and twisted photon (bottom) with opening angle $\theta_{p}=30^{\circ}$. The organization of the figure and all other parameters are the same as for Fig. 8 .
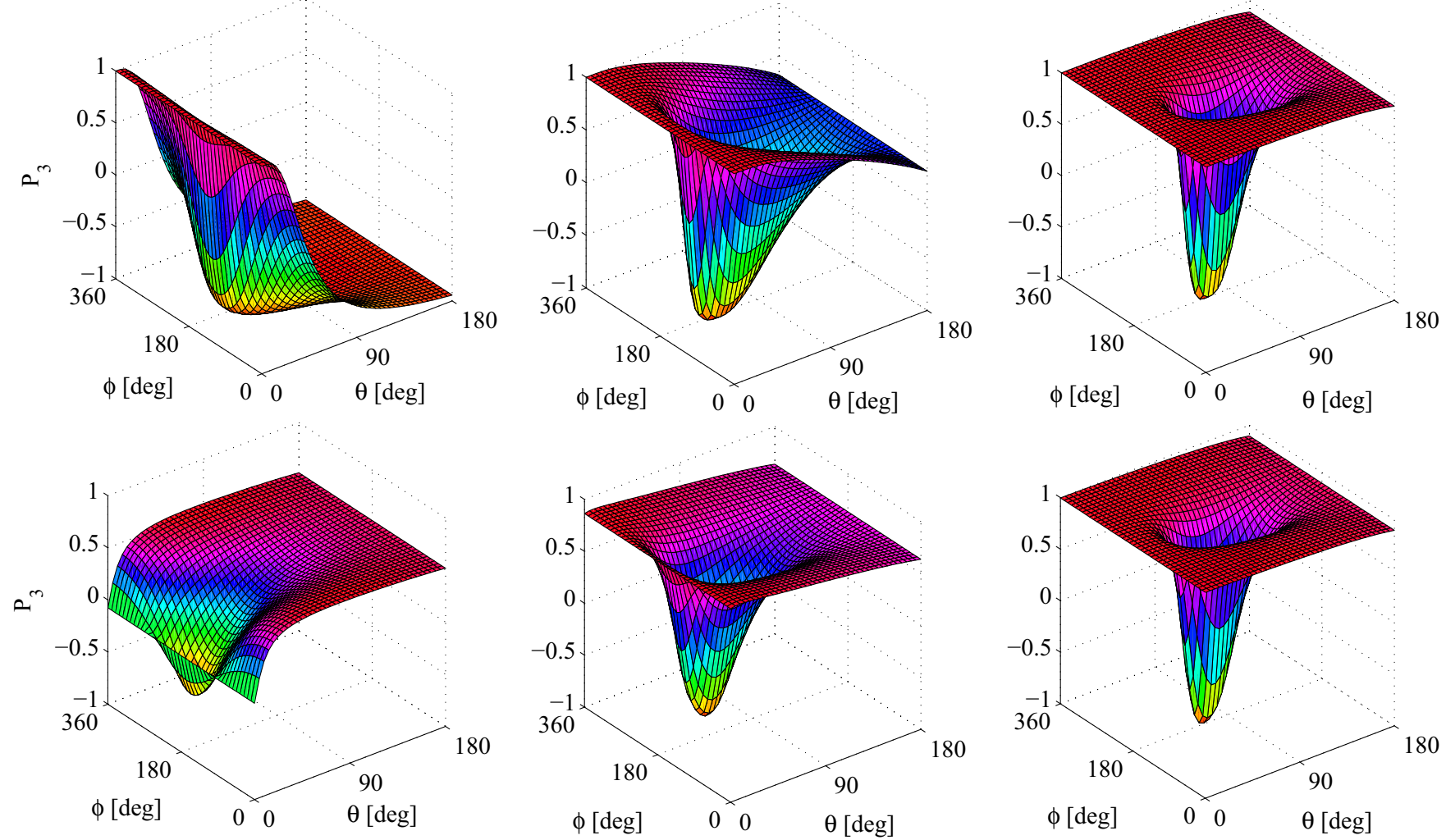

FIG. 10. Stokes parameter $P_{3}$ as a function of the scattering angles $\theta$ and $\phi$ for an incident plane-wave photon (top row) and twisted photon (bottom row) with opening angle $\theta_{p}=30^{\circ}$. The organization of the figure and all other parameters are the same as for Fig. 8 . 
relative sign difference between the two distributions in any given direction of scattering has largely disappeared. Finally, for $\theta_{i}=60^{\circ}$ (third column), the distributions are even more alike.

\section{The inversion cone}

In this section, we develop a criterion by which to estimate the maximum value of $\theta_{i}$ inside which $P_{3}$ from twisted and plane-wave photons tend to have opposite signs. To this end, Eq. (32) is recast. Instead of referring to the fixed $z$ axis in the laboratory frame, the kinematics is referred to the direction of motion of the electron (i.e., $\vec{n}_{i}$ ) with the replacement $\beta_{z} \rightarrow$ $\left|\vec{\beta}_{i}\right|=\beta_{i}$. The unit vector $\vec{n}_{i}$ defines a $\zeta \zeta^{\prime}$ axis common to the laboratory frame and rest frame of the electron. Relative to this direction, a plane-wave photon, or plane-wave component of a twisted photon, which is oriented at angle $\vartheta$ (given by $\cos \vartheta=\vec{n}_{0} \cdot \vec{n}_{i}$ ) to the electron in the laboratory frame, will map into angle $\vartheta^{\prime}$ in the rest frame of the electron. Hence, making the additional replacements $\theta_{p} \rightarrow \vartheta$ and $\theta_{p}^{\prime} \rightarrow \vartheta^{\prime}$ in Eq. (32), imposing the condition for the threshold of inversion in the electron rest frame $\cos \vartheta^{\prime}=0$, and solving for $\cos \vartheta$ yields

$$
\cos \vartheta_{I}=\beta_{i},
$$

where the inversion angle $\vartheta_{I}$ can be interpreted as the opening angle of a cone of rays swept out about the direction of the electron motion $\vec{n}_{i}$ in the laboratory frame. If the direction of a plane-wave photon or plane-wave component of a twisted photon (i.e., $\vec{n}_{0}$ ) falls outside of this so-called inversion cone (i.e., $\vartheta>\vartheta_{I}$ ) then it will have turned around to some degree in a backward facing direction (measured relative to the common $\zeta \zeta^{\prime}$ axis) in the rest-frame of the electron (in other words, in the laboratory frame $\cos \vartheta>0$ but in the electron rest frame $\cos \vartheta^{\prime}<0$ ), otherwise it will not.

Let us reanalyze the case $\theta_{i}=0^{\circ}$ in which the electron and photon are both traveling along the $z$ direction. In this case, $\beta_{x}=\beta_{y}=0$ and $\beta_{z}=\left|\vec{\beta}_{i}\right|=\beta_{i}$. Hence, for $\beta_{z}<\beta_{z}^{c}$, the momentum cone of the twisted photon falls entirely within the inversion cone, as would a plane-wave photon moving along the positive $z$ direction. No momentum-cone inversion occurs, and there should be no relative sign flip in the distribution of $P_{3}$ for the twisted photon as compared to that for the plane-wave photon. As $\beta_{i}$ increases, the opening angle $\vartheta_{I}$ decreases. For $\beta_{i}=\beta_{z}>\beta_{z}^{c}$, the inversion cone angle satisfies $\vartheta_{I}<\theta_{p}$, and the entire momentum cone of the twisted photon inverts $\left(\cos \vartheta^{\prime}<0\right)$ in the electron rest frame. For a planewave photon traveling along positive $z$, its direction will be unaffected. Therefore, in this case a sign flip of $P_{3}$ from the twisted photon for a wide range of $\theta$ is expected as compared to that from the plane-wave photon, as discussed in relation to the results of Fig. 6 .

Next we consider the scenario where $\beta_{i}>\beta_{z}^{c}$ as $\theta_{i}>0^{\circ}$ increases. As $\theta_{i}$ increases the inversion cone also rotates by $\theta_{i}$ away from the $z$ direction. Furthermore, since it is assumed that $\beta_{i}>\beta_{z}^{c}$, the inversion cone opening angle $\vartheta_{I}$ is smaller than the momentum cone opening angle $\theta_{p}$ of the twisted photon. If $\theta_{i}$ is sufficiently small, even as it rotates the inversion cone will remain entirely (or predominately) within the momentum cone of the twisted photon, but the momentum of a plane-wave photon propagating along positive $z$ direction will still remain within the inversion cone. If these conditions are met, namely,

$$
\theta_{i}<\vartheta_{I}<\theta_{p},
$$

where the second inequality also incorporates the condition $\beta_{i}>\beta_{z}^{c}$, then the distribution for $P_{3}$ from the scattering of a twisted photon will undergo a sign shift over a wide range of $\theta$ relative to the distribution from a plane-wave photon. These were precisely the conditions attained for the distributions in the first column of Fig. 10. For the twisted photon and electron considered there, $\theta_{i}=10^{\circ}$, whereas $\vartheta_{I} \approx 22.9^{\circ}$ for the value of $T_{i}$ chosen, and $\theta_{p}=30^{\circ}$.

As $\theta_{i}$ continues to increase such that the first relation in Eq. (35) is violated, even if the second is not, a plane-wave photon propagating along the positive $z$ direction will now fall outside the inversion cone too. Consequently, the relative sign shift between twisted and plane-wave photons should disappear over most scattering directions. This is precisely the situation displayed in the second and third columns of Fig. 10, with the distributions for twisted and plane-wave photons displaying increasingly similar behavior for most scattering angles.

\section{E. Scattering of a photon in a superposition of twisted Bessel states}

The differential cross section for a particle prepared in a pure Bessel state that scatters from another particle prepared in a plane-wave state is only sensitive to the cone opening angle $\theta_{p}$ and not on the projection of angular momentum $m$ [43] of the twisted particle. This lack of dependence partly motivates the theoretical $[24,27,43,53]$ investigation of various scattering processes with a superposition of twisted states. As these and other studies have shown, the angular distributions of the scattered particles still do not depend on the specific values of angular momentum projection $m_{1}$ or $m_{2}$ individually. Rather, they depend on the difference $\Delta m=$ $m_{2}-m_{1}$ of these values.

Likewise, there is a parallel motivation for the experimental realization of photons with a superposition of twisted states, which is also progressing. Early efforts [54] focused on the preparation of optical photons. However, by adapting the diffraction optics used to generate twisted electrons, recent experiments at the Advanced Light Source [49] report on the development of special diffraction gratings that can directly generate superposition states of twisted soft $\mathrm{x}$ rays from a beam operating between $400 \mathrm{eV}$ and $1.3 \mathrm{keV}$. Another recent study [50] reports on the development of specially designed spiral phase plates to create hard x-ray beams with an energy of $8.2 \mathrm{keV}$ carrying orbital angular momentum, whereas another group [48] reports on the direct generation of Bessel beams up to $15 \mathrm{keV}$ with a refractive $\mathrm{x}$-ray axicon. These studies suggest that further progress in $\mathrm{x}$-ray optics will make it possible to produce superpositions of twisted beams of moderately high energy $(\sim 100 \mathrm{keV})$ in the future.

Returning in this section to the case whereby the electron moves strictly in either the positive or negative $z$ direction, we consider Compton scattering of a photon prepared in a coherent superposition of two twisted Bessel states, each of 

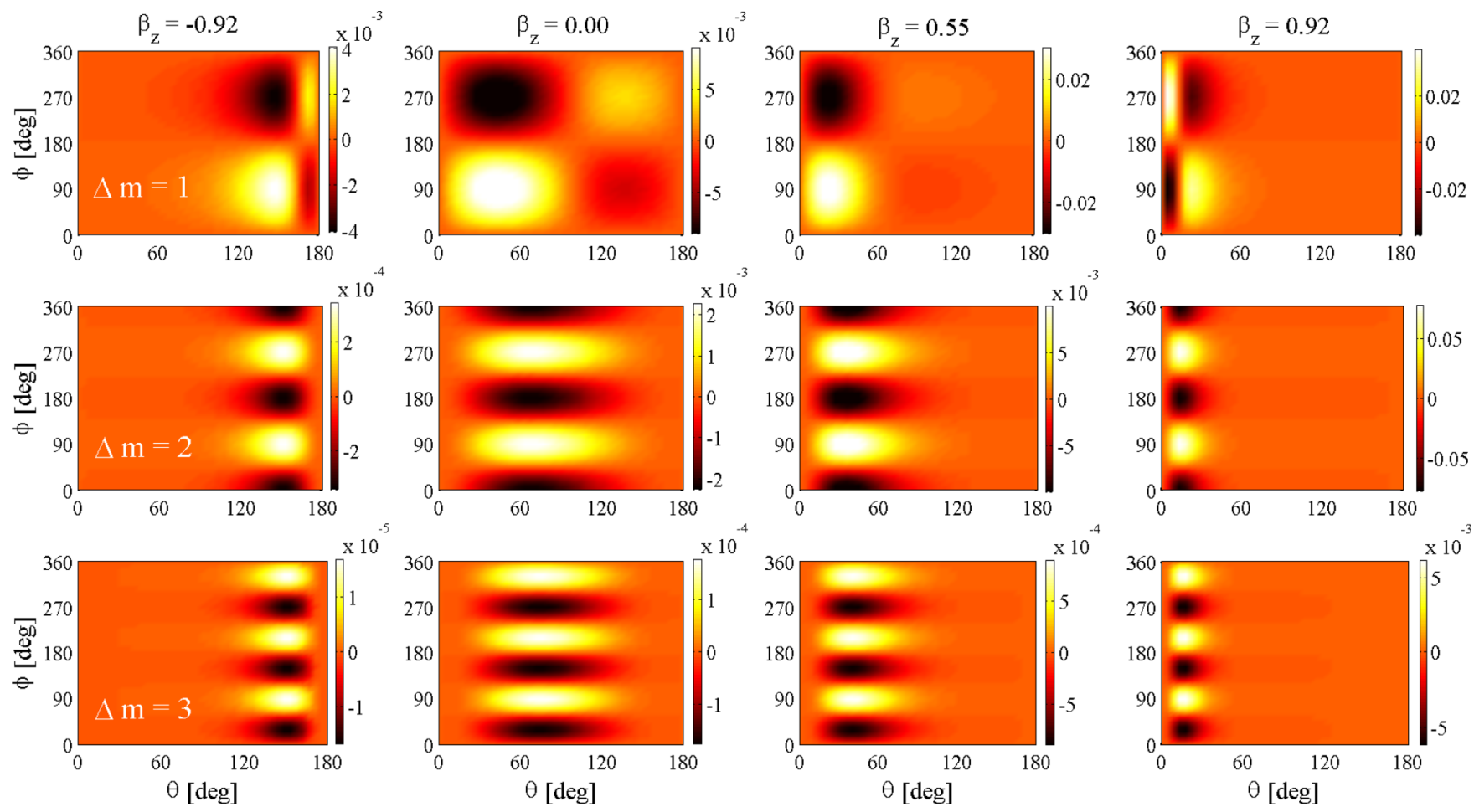

FIG. 11. Interference differential cross section $\left(\frac{d \sigma_{2 \mathrm{tw}}}{d \Omega}\right)_{\mathrm{int}}=\frac{d \sigma_{2 \mathrm{tw}}}{d \Omega}-\frac{d \sigma_{\mathrm{tw}}}{d \Omega}$ plotted as a function of the scattering angles $\theta$ and $\phi$ for selected values of $\Delta m$ of the photon and dimensionless velocity component $\beta_{z}$ of the electron, while $\beta_{x}=\beta_{y}=0$. For the twisted photon, which is unpolarized, $E_{0}=100 \mathrm{keV}, \theta_{p}=30^{\circ}$. (Top) $\Delta m=1$, (middle) 2 , and (bottom) 3 . The color-coded scale for all panels is in units of b/sr.

which has the same transverse and longitudinal momenta $\kappa_{p_{\perp}}$ and $\kappa_{p_{\|}}$and equal helicity $\Lambda$, but with different values of total angular momentum projection $m_{1}$ and $m_{2}>m_{1}$. The state vector for a photon prepared this way is of the form

$$
|\gamma\rangle=c_{1}\left|\kappa_{p_{\perp}} \kappa_{p_{\|}} m_{1} \Lambda\right\rangle+c_{2}\left|\kappa_{p_{\perp}} \kappa_{p_{\|}} m_{2} \Lambda\right\rangle
$$

where the coefficients satisfy $\left|c_{1}\right|^{2}+\left|c_{2}\right|^{2}=1$ and the state $|\gamma\rangle$ is normalized $\langle\gamma \mid \gamma\rangle=1$. One choice for the $c_{n}$, used in this paper, is $c_{1}=1 / \sqrt{2}$ and $c_{2}=e^{i \delta} / \sqrt{2}$. These coefficients are of equal modulus and therefore each twisted state in the superposition is weighted equally. Hence, this superposition state can be described by a modified version of Eq. (6). The amplitude given by Eq. (8) is replaced by the modified transverse amplitude [43]

$$
A_{\kappa_{p_{\perp}} m_{1} m_{2}}\left(\vec{p}_{0_{\perp}}\right)=\frac{1}{\sqrt{2}}\left[a_{\kappa_{p_{\perp}} m_{1}}\left(\vec{p}_{0_{\perp}}\right)+e^{i \delta} a_{\kappa_{p_{\perp}} m_{2}}\left(\vec{p}_{0_{\perp}}\right)\right] \text {, }
$$

where the plane-wave components are no longer weighted uniformly with respect to the azimuthal angle $\phi_{p}$. This new amplitude modifies Eq. (11) for a pure twisted Bessel state to a modified form for a superposition of twisted Bessel states given by [53]

$$
\frac{d \sigma_{2 \mathrm{tw}}}{d \Omega}=\int \frac{d \phi_{p}}{2 \pi} F\left(\phi_{p}\right) \frac{d \sigma_{\mathrm{pw}}\left(\theta_{p}, \phi_{p}\right)}{d \Omega},
$$

where the azimuthal distribution

$$
F\left(\phi_{p}\right)=1+\cos \left[\Delta m\left(\phi_{p}-\frac{\pi}{2}\right)+\delta\right],
$$

depends on the difference of the projection numbers, $\Delta m=$ $m_{2}-m_{1}$. In this study, the integrand of Eq. (38) is given by Eq. (24) for a sum-averaged cross section. Also, in this section, the electron is assumed to move along the $z$ axis (as does the twisted photon) and the flux factors present in Eq. (11) have canceled in Eq. (38).

Finally, as is clear from Eqs. (38) and (39), the cross section for the superposition of two Bessel states can be viewed as the sum of the cross section for the pure Bessel state and an interference term which varies with $\phi_{p}$ and $\Delta m$. It is this interference term which contains the interesting new features. Unfortunately, this term tends to be small compared to the one corresponding to the pure Bessel state. To clarify the effects of using a superposition of Bessel states, we focus on presenting the interference term given by

$$
\left(\frac{d \sigma_{2 \mathrm{tw}}}{d \Omega}\right)_{\mathrm{int}}=\frac{d \sigma_{2 \mathrm{tw}}}{d \Omega}-\frac{d \sigma_{\mathrm{tw}}}{d \Omega},
$$

where $\left(\frac{d \sigma_{2 \text { tw }}}{d \Omega}\right)_{\text {int }}$ is the interference part of the cross section for a superposition Bessel state.

In Fig. 11, the interference differential cross section $\left(\frac{d \sigma_{2 \mathrm{tw}}}{d \Omega}\right)_{\text {int }}$ is plotted as a function of the scattering angles $\theta$ and $\phi$ for selected values of $\Delta m$ of the photon and dimensionless velocity component $\beta_{z}$ of the electron, while its other components are $\beta_{x}=\beta_{y}=0$. The photon is prepared in a coherent superposition of two equally weighted, unpolarized Bessel states of energy $E_{0}=100 \mathrm{keV}$, with cone opening angle $\theta_{p}=30^{\circ}$.

The results for an electron at rest $\left(\beta_{z}=0\right)$ are shown in the second column of panels in the figure. The main features for this case are the following. The number of azimuthal modulations is $\Delta m$, and for each increment in $\Delta m$, the relative size of these modulations decreases significantly. Finally, for 
$\Delta m=1$, there is an additional polar variation. These results are in agreement with those reported [24,25] in previous studies concerning twisted photons scattering from electrons at rest. Additionally, we note that for this case, the azimuthal modulations consist of one which is primarily confined in the upper hemisphere (i.e., $\theta \leqslant 90^{\circ}$ ) and the other one which is confined in the lower hemisphere. Henceforth we shall refer to these as the small-polar azimuthal modulation and the largepolar azimuthal modulation, respectively. This terminology will be used even for the cases whereby the electron is in motion and the modulations shift location with respect to the polar angle.

The results for which the electron and twisted photon undergo a head-on collision are displayed in the first column of panels in the figure corresponding to $\beta_{z}=-0.92$. The strong backscattering of the photon for this case is reflected in the significant shift of the modulations toward larger values of $\theta$ and their compression into a smaller solid angle as compared to the electron at rest. Moreover, regardless of the value of $\Delta m$ the modulations in this case are significantly smaller in magnitude than those corresponding to $\beta_{z}=0$. This result is consistent with the opening angle of the twisted photon becoming smaller, for this case, in the rest frame of the electron.

In the third column of panels in the figure, corresponding to $\beta_{z}=0.55$, results are shown for an electron and twisted photon undergoing a same-direction collision. Here strong forward scattering is reflected by the significant shift of the modulations toward smaller values of $\theta$ as compared to those for an electron at rest. Furthermore, these modulations tend to be significantly larger for any value of $\Delta m$ as compared to those for an electron at rest, which reflects the enhanced effects arising from the increased opening angle of the twisted photon in the electron rest frame.

The fourth column of panels in the figure display the results for $\beta_{z}=0.92$. Most of the behavior of these results is similar to that in the third column, except for those corresponding to $\Delta m=1$. In this case, the modulations behave differently according to the signs of their corresponding lobes. For example, if the electron is at rest, the small-polar azimuthal modulation has a positive lobe within $0<\phi<180^{\circ}$ followed by a negative lobe within $180^{\circ}<\phi<360^{\circ}$, while the largepolar azimuthal modulation, which is somewhat smaller in magnitude, has a negative lobe followed by a positive one in the same corresponding ranges of azimuthal angles. The results for which $\beta_{z}=-0.92$ and 0.55 also retain this ordering with respect to the sign of the lobes for both the smallpolar and large-polar azimuthal modulations, even though the motion of the electron causes them to shift with respect to their polar-angle location and to distort in relation to the electronat-rest case. Parenthetically, for the case where $\beta_{z}=0.55$, the large-polar azimuthal modulation is present, but relativistic effects have spread it out over a large solid angle, and it is consequently very faint. For the case $\beta_{z}=0.92$, on the other hand, the small-polar azimuthal modulation now has a negative lobe followed by a positive one, and the large-polar modulation has a positive lobe followed by a negative one. That is, the signs of the lobes are in the reverse order to those for the other cases.
This behavior can be understood by considering that for opening angle $\theta_{p}=30^{\circ}$, the critical velocity is $\beta_{z}^{c} \approx 0.87$. The case $\beta_{z}=0.92$ satisfies the condition $\beta_{z}>\beta_{z}^{c}$, but the values of $\beta_{z}$ for the other cases do not. Physically, when $\beta_{z}>\beta_{z}^{c}$, the momentum cone of the twisted photon inverts $\left(\cos \theta_{p}^{\prime}<0\right)$ in the rest frame of the electron. This essentially reverses the roles of forward and backward scattering in that frame. In the laboratory frame, this implies that the identities for the small-polar and large-polar modulations are the reverse to those cases for which $\beta_{z}<\beta_{z}^{c}$.

For a twisted photon in a superposition state of the same energy but much smaller opening angle than for the distributions presented in Fig. 11, the results are similar, except the magnitude of the modulations tend to have smaller peak values than corresponding distributions of the twisted photon with larger opening angle. Further calculations on a $100 \mathrm{keV}$ twisted photon with opening angle of $\theta_{p}=3^{\circ}$ for several choices of superposition state were performed. When the electron is at rest and $\Delta m=1$, for example, the results are almost identical to the corresponding panel in Fig. 11 except the peak magnitude of the modulations is smaller by approximately a factor of five than those shown, and for the electron at rest and $\Delta m=3$, the peak magnitude is approximately five hundred times smaller. As for the case with larger opening angles, scattering from an electron with $\beta_{z}>0$ increases the size of the modulation peak magnitude, and scattering from an electron with $\beta_{z}<0$ decreases it. Similarly, the modulations undergo a reversal when $\beta_{z}>\beta_{z}^{c}$ and $\Delta m=1$. In this case the critical velocity $\beta_{z}^{c} \approx 0.998$, or $T_{i}^{c} \approx 9.25 \mathrm{MeV}$, for $\theta_{p}=$ $3^{\circ}$. When $T_{i}=14 \mathrm{MeV}$, calculations indicate that the peak magnitude of the modulation is roughly twenty times greater than for the panel $\left(\beta_{z}=0.92, \Delta m=1\right)$ corresponding to modulation reversal in Fig. 11.

Finally, we consider what happens for the case of lowenergy photons in the Thomson limit. Further calculations were performed on a $10 \mathrm{eV}$ twisted photon with the opening angle $\theta_{p}=30^{\circ}$ and for the same choices of $\beta_{z}$ as for the $100 \mathrm{keV}$ twisted photon in Fig. 11. The calculations indicate that for $\Delta m=1$ and $\Delta m=2$ the distributions are similar in appearance and comparable in magnitude to those for a 100 $\mathrm{keV}$ twisted photon, as shown in the corresponding rows in Fig. 11. There is also a reversal in the sign of the modulation for the distribution corresponding to $\Delta m=1$ and $\beta_{z}=0.92$, similar to the reversal displayed by the corresponding distribution of a $100 \mathrm{keV}$ twisted photon. However, identical calculations on a $10 \mathrm{eV}$ twisted photon for $\Delta m \geqslant 3$ reveal negligible variation of the distributions in comparison to those for $\Delta m=2$, in agreement with the results reported in a previous study [24] on the scattering of a low-energy twisted photon from an electron at rest. There, the difference in behavior was attributable to the operation of dipole selection rules in the Thomson-scattering limit. Here, the dipole selection rules work in conjunction with an interplay between the Doppler effect and the effect which transforms the opening angle. That is, the condition (head-on collision) which increases the energy of the photon from the laboratory frame to the rest frame of the electron also reduces the opening angle. The condition (same-direction collision) which increases the opening angle further reduces the energy of the low-energy photon, 
enforcing the selection rules regardless of the increased size of the opening angle.

\section{SUMMARY AND CONCLUSION}

In summary, we have studied Compton scattering of a twisted Bessel photon by a plane-wave electron within the framework of quantum electrodynamics. Specifically, we examined the extent to which the motion of the electron affects the dependence of the angular distributions of the sum-averaged cross section and the Stokes parameters on the twisted photon momentum cone opening angle and the projection of its intrinsic total angular momentum.

In particular, we found that the electron motion influences the dependence of the distributions on the opening angle of the twisted photon, but this influence differs depending on the direction of motion of the electron. In a head-on collision, for example, the electron motion diminishes the effects of the opening angle of the twisted photon on the distributions, but in a same-direction collision, which is a process significantly different from the plane-wave case, the electron motion enhances them. Moreover, if the speed of the electron exceeds a specific critical value, which depends on the momentum-cone opening angle of the twisted photon, during a same-direction collision, then certain distributions, such as for Stokes parameter $P_{3}$, will undergo a change of sign over a large range of the scattering angles as compared to their corresponding value for which a plane-wave photon is scattered. This indicates, for example, that the helicity of the scattered photon tends to be the reverse of the usual case. A similar sign reversal was found to occur for the $\Delta m=1$ angular distribution of the interference term of the superposition Bessel state differential cross section. Ultimately, this behavior corresponds to the inversion of the momentum cone of the twisted photon in the rest frame of the electron.

Moreover, although the results were shown to be sensitive to differences of the projection of total angular momentum $\Delta m$ for a photon prepared in a superposition of pure Bessel states, they remain insensitive to any specific value of $m$. This insensitivity to $m$ also holds for the scattering of photons in pure Bessel states. This behavior is due primarily to the wave function of the electron being approximated as a plane wave, which has infinite transverse extent. Sensitivity to $m$ is expected to reappear if the transverse spatial extent of the electron target is small (i.e., $\lesssim \hbar / \kappa_{p_{\perp}}$ ). However, the effects associated with this scenario are outside the scope of the present study and will be presented elsewhere. Otherwise, even for an electron with a realistic wave function of finite spatial dimensions, if its momentum is sufficiently sharp and its transverse spatial extent is large (i.e., $\gg \hbar / \kappa_{p_{\perp}}$ ), the numerical results will reduce to those presented in this study. Finally, we point out that some of the results presented in this study, such as the effects arising with regard to momentum-cone inversion, need not wait for the realization of high-energy twisted Bessel photons for their experimental exploration but should be accessible to those available currently or that will become available in the near future.
[1] L. Allen, M. W. Beijersbergen, R. J. C. Spreeuw, and J. P. Woerdman, Phys. Rev. A 45, 8185 (1992).

[2] M. W. Beijersbergen, L. Allen, H. E. L. O. van der Veen, and J. P. Woerdman, Opt. Commun. 96, 123 (1993).

[3] G. Molina-Terriza, J. P. Torres, and L. Torner, Nat. Phys. 3, 305 (2007).

[4] The Angular Momentum of Light, edited by D. L. Andrews and M. Babiker (Cambridge University Press, Cambridge, U.K., 2013).

[5] S. M. Barnett and L. Allen, Opt. Commun. 110, 670 (1994).

[6] L. Allen, M. Padgett, and M. Babiker, Progress in Optics (Elsevier, Amsterdam, 1999), Vol. 39, pp. 291-372.

[7] A. M. Yao and M. J. Padgett, Adv. Opt. Photonics 3, 161 (2011).

[8] X. Cai, J. Wang, M. J. Strain, B. Johnson-Morris, J. Zhu, M. Sorel, J. L. O'Brien, M. G. Thompson, and S. Yu, Science 338, 363 (2012).

[9] N. Bozinovic, Y. Yue, Y. Ren, M. Tur, P. Kristensen, H. Huang, A. E. Willner, and S. Ramachandran, Science 340, 1545 (2013).

[10] H. He, M. E. J. Friese, N. R. Heckenberg, and H. RubinszteinDunlop, Phys. Rev. Lett. 75, 826 (1995).

[11] M. Padgett and R. Bowman, Nat. Photonics 5, 343 (2011).

[12] A. Jesacher, S. Furhapter, S. Bernet, and M. Ritsch-Marte, Phys. Rev. Lett. 94, 233902 (2005).

[13] A. Afanasev, C. E. Carlson, and A. Mukherjee, Phys. Rev. A 88, 033841 (2013).

[14] H. M. Scholz-Marggraf, S. Fritzsche, V. G. Serbo, A. Afanasev, and A. Surzhykov, Phys. Rev. A 90, 013425 (2014).
[15] A. Afanasev, C. E. Carlson, and A. Mukherjee, J. Opt. 18, 074013 (2016).

[16] A. Surzhykov, D. Seipt, V. G. Serbo, and S. Fritzsche, Phys. Rev. A 91, 013403 (2015).

[17] L. Kaplan and J. H. McGuire, Phys. Rev. A 92, 032702 (2015).

[18] A. Afanasev, C. E. Carlson, and M. Solyanik, Phys. Rev. A 97, 023422 (2018).

[19] C. Schmiegelow and F. Schmidt-Kaler, Eur. Phys. J. D 66, 157 (2012).

[20] C. T. Schmiegelow, J. Schulz, H. Kaufmann, T. Ruster, U. G. Poschinger, and F. Schmidt-Kaler, Nat. Commun. 7, 12998 (2016).

[21] A. Afanasev, C. E. Carlson, C. T. Schmiegelow, J. Schulz, F. Schmidt-Kaler, and M. Solyanik, New J. Phys. 20, 023032 (2018).

[22] A. Picón, J. Mompart, J. R. Vázquez de Aldana, L. Plaja, G. F. Calvo, and L. Roso, Opt. Express 18, 3660 (2010).

[23] A. A. Peshkov, A. V. Volotka, A. Surzhykov, and S. Fritzsche, Phys. Rev. A 97, 023802 (2018).

[24] S. Stock, A. Surzhykov, S. Fritzsche, and D. Seipt, Phys. Rev. A 92, 013401 (2015).

[25] J. A. Sherwin, Phys. Rev. A 96, 062120 (2017).

[26] T. Maruyama, T. Hayakawa, and T. Kajino, Sci. Rep. 9, 51 (2019).

[27] J. A. Sherwin, Phys. Rev. A 95, 052101 (2017).

[28] A. Afanasev, V. G. Serbo, and M. Solyanik, J. Phys. G 45, 055102 (2018). 
[29] Y. Taira, T. Hayakawa, and M. Katoh, Sci. Rep. 7, 5018 (2017).

[30] G. Gariepy, J. Leach, K. T. Kim, T. J. Hammond, E. Frumker, R. W. Boyd, and P. B. Corkum, Phys. Rev. Lett. 113, 153901 (2014).

[31] C. Hernández-García, J. San Román, L. Plaja, and A. Picón, New J. Phys. 17, 093029 (2015).

[32] R. Géneaux, A. Camper, T. Auguste, O. Gobert, J. Caillat, R. Taeib, and T. Ruchon, Nat. Commun. 7, 12583 (2016).

[33] S. Sasaki and I. McNulty, Phys. Rev. Lett. 100, 124801 (2008).

[34] E. Hemsing, A. Marinelli, and J. B. Rosenzweig, Phys. Rev. Lett. 106, 164803 (2011).

[35] O. V. Bogdanov, P. O. Kazinski, and G. Y. Lazarenko, Phys. Rev. A 97, 033837 (2018).

[36] U. D. Jentschura and V. G. Serbo, Phys. Rev. Lett. 106, 013001 (2011).

[37] U. D. Jentschura and V. G. Serbo, Eur. Phys. J. C 71, 1571 (2011).

[38] V. Petrillo, G. Dattoli, I. Drebot, and F. Nguyen, Phys. Rev. Lett. 117, 123903 (2016)

[39] C. Liu, B. Shen, X. Zhang, Y. Shi, L. Ji, W. Wang, L. Yi, L. Zhang, T. Xu, Z. Pei, and Z. Xu, Phys. Plasmas 23, 093120 (2016).

[40] Y. Taira and M. Katoh, Astrophys. J. 860, 45 (2018).

[41] F. Tamburini, E. Mari, B. Thidé, G. Molina-Terriza, and G. Anzolin, Nat. Phys. 7, 195 (2011)
[42] K. Y. Bliokh, I. P. Ivanov, G. Guzzinati, L. Clark, R. Van Boxem, A. Béché, R. Juchtmans, M. A. Alonso, P. Schattschneider, F. Nori, and J. Verbeeck, Phys. Rep. 690, 1 (2017).

[43] I. P. Ivanov, Phys. Rev. D 83, 093001 (2011).

[44] M. Kaku, Quantum Field Theory: A Modern Introduction (Oxford University Press, New York, 1993).

[45] M. E. Peskin and D. V. Schroeder, An Introduction to Quantum Field Theory (Westview, Boulder, CO, 1995).

[46] G. A. Krafft, E. Johnson, K. Deitrick, B. Terzić, R. Kelmar, T. Hodges, W. Melnitchouk, and J. R. Delayen, Phys. Rev. Accel. Beams 19, 121302 (2016).

[47] H. Mimura et al., Nat. Phys. 6, 122 (2010).

[48] D. Zverev, A. Barannikov, I. Snigireva, and A. Snigirev, Opt Express 25, 28469 (2017).

[49] J. C. T. Lee, S. J. Alexander, S. D. Kevan, S. Roy, and B. J. McMorran, Nat. Photon. 13, 205 (2019).

[50] F. Seiboth, M. Kahnt, M. Lyubomirskiy, M. Seyrich, F. Wittwer, T. Ullsperger, S. Nolte, D. Batey, C. Rau, and C. G. Schroer, Opt. Lett. 44, 4622 (2019).

[51] D. Giovannini, J. Romero, V. Potoček, G. Ferenczi, F. Speirits, S. M. Barnett, D. Faccio, and M. J. Padgett, Science 347, 6224 (2015).

[52] P. Saari, Phys. Rev. A 97, 063824 (2018).

[53] D. Seipt, A. Surzhykov, and S. Fritzsche, Phys. Rev. A 90 , 012118 (2014).

[54] R. Vasilyeu, A. Dudley, N. Khilo, and A. Forbes, Opt. Express 17, 23389 (2009). 